\title{
COMPOSIÇÃO FLORÍSTICA E ANÁLISE FITOGEOGRÁFICA DE UMA FLORESTA SEMIDECÍDUA Na BAHIA, BRASIL
}

\author{
Domingos Benício Oliveira Silva Cardoso ${ }^{1}$, Flávio França ${ }^{1}$, Jaílson Santos de \\ Novais $^{2}$, Marcio Harrison dos Santos Ferreira ${ }^{1}$, Rubens Manoel dos Santos ${ }^{3}$, \\ Vinícius Mendes Souza Carneiro ${ }^{1}$ \& Jacqueline Miranda Gonçalves ${ }^{1}$
}

\section{RESUMO}

(Composição florística e análise fitogeográfica de uma floresta semidecídua na Bahia, Brasil) As florestas semidecíduas na Bahia mostram-se bastante fragmentadas e têm sido pouco estudadas em relação à florística e à fitogeografia. Estas florestas estão situadas principalmente no semi-árido e isoladas da Mata Atlântica costeira por extensas áreas de caatinga. Este trabalho apresenta o levantamento florístico de um fragmento de floresta semidecídua na Serra da Fazenda Retiro (SFR), localizada no município de Feira de Santana, Bahia, com o objetivo principal de avaliar a sua posição fitogeográfica em relação às caatingas e à Mata Atlântica. Foram amostradas 173 espécies incluídas em 143 gêneros e 59 famílias. Myrtaceae foi a família que apresentou o maior número de espécies (15 spp.), seguida por Euphorbiaceae (13), Leguminosae (12), Malvaceae (7), Orchidaceae (7) e Rubiaceae (7). Além disso, foram coletadas três novas espécies dos gêneros Neomarica (Iridaceae), Pseudobombax (Malvaceae) e Solanum (Solanaceae). As análises de agrupamento pelo método UPGMA e de composição de espécies da SFR mostram que a área, apesar de estar situada dentro do Bioma Caatinga, apresenta uma maior relação florística com as florestas semidecíduas do Domínio da Floresta Atlântica.

Palavras-chave: fitogeografia, flora, florestas secas, Mata Atlântica, semi-árido.

\section{Abstract}

(Floristic composition and phytogeographical analysis of a semideciduous forest in Bahia State, Brazil) The semideciduous forests of Bahia occur as highly fragmented areas and have only received a little attention from floristic and phytogeographical studies. These areas are mainly situated within the semi-arid region and isolated from the Coastal Atlantic Forest by the surrounding dry vegetation of the caatinga domain. This paper presents a floristic survey of a semideciduous forest fragment at Serra da Fazenda Retiro (SFR), Feira de Santana, Bahia State, in order to assess its phytogeographical relationships in comparison to caatinga vegetation and Atlantic Forest. The survey identified 173 species belonging to 143 genera and 59 families. Myrtaceae presented the highest species number (15 spp.), followed by Euphorbiaceae (13), Leguminosae (12), Malvaceae (7), Orchidaceae (7), and Rubiaceae (7). Additionally, three new species of the genera Neomarica (Iridaceae), Pseudobombax (Malvaceae), and Solanum (Solanaceae) were sampled. In spite of the studied area being located within the circumscription of the Caatinga Biome, analysis of similarity (UPGMA) and of the SFR species composition showed that it is more floristically related to semideciduous forest of the Atlantic Forest domain rather than to caatinga vegetation.

Key words: phytogeography, flora, dry forests, Atlantic Forest, semi-arid.

\section{INTRODUÇão}

O Bioma Caatinga é o tipo de vegetação estacional que cobre a maior parte da área com clima semi-árido da região Nordeste do Brasil (Rodal \& Sampaio 2002; Velloso et al. 2002; Prado 2003), principalmente em altitudes mais baixas, entre planaltos e serras, compreendendo uma área aproximada de $800.000 \mathrm{~km}^{2}$ (Ab'Saber
1974). De modo geral, as caatingas têm sido caracterizadas fisionomicamente como florestas de porte baixo, compreendendo árvores e arbustos que comumente apresentam espinhos e microfilia (Rizzini 1997; Prado 2003; Queiroz 2006). Poroutro lado, Andrade-Lima (1981) considera que as caatingas incluem também áreas onde predomina vegetação arbórea. Ainda não existe um consenso

Artigo recebido em 05/2009. Aceito para publicação em 09/2009.

${ }^{1}$ Universidade Estadual de Feira de Santana, Depto. Ciências Biológicas, Av. Transnordestina s/n, Novo Horizonte, 44036-900, Feira de Santana, BA. Autor para correspondência: cardosobot@ gmail.com

${ }^{2}$ Instituto Nacional de Pesquisas da Amazônia, Coordenação de Pesquisas em Botânica, Lab. Palinologia, Av. André Araújo 2936, C.P. 478, Aleixo, 69060-001, Manaus, AM.

${ }^{3}$ Universidade Federal de Lavras, Depto. Engenharia Florestal, 37200-000, Lavras, MG. 
quanto à delimitação e à classificação fitogeográfica dessas áreas, principalmente por não serem homogêneas (Veloso et al. 1991), estando sujeitas a variações locais de substrato, fatores climáticos e topografia. Alguns autores preferem denominar essas florestas altas de caatinga como florestas estacionais (Rizzini 1997; Veloso et al. 1991). Tais florestas estacionais ocorrem em faixas descontínuas e estão fortemente marcadas pela influência de uma estação chuvosa curta e uma estação seca mais prolongada. Esta característica climática é também responsável pela caducifolia de grande parte das árvores em resposta ao período de deficiência hídrica (Murphy \& Lugo 1986). Padrões de distribuição disjunta entre as áreas descontínuas de florestas secas neotropicais, além do clima marcadamente sazonal, foram determinantes para incluir as florestas estacionais brasileiras, juntamente com as caatingas, dentro do Domínio das Florestas Tropicais Sazonalmente Secas (Prado \& Gibbs 1993; Prado 2000; Pennington et al. 2000, 2006). Em termos fitogeográficos, Melo \& Rodal (2003) também afirmam que as florestas estacionais são caracterizadas como uma vegetação de transição entre a Floresta Atlântica e as caatingas, ocupando uma estreita faixa entre esses dois Domínios vegetacionais. No entanto, Oliveira-Filho \& Fontes (2000), estudando os padrões de distribuição de espécies arbóreas entre diferentes fisionomias florestais do sudeste e centro-oeste do Brasil, e OliveiraFilho et al. (2006), analisando a similaridade florística entre diversas áreas do leste do Brasil, propuseram que as florestas estacionais deveriam fazer parte do Domínio da Floresta Atlântica. Particularmente em relação a algumas florestas estacionais da Bahia, esta posição também foi defendida por Amorim et al. (2005a), Oliveira-Filho et al. (2005) e Cardoso \& Queiroz (2008).

A maior parte dos estudos florísticos no Nordeste tem dado ênfase, sobretudo, à vegetação fanerogâmica lenhosa (e.g. Rodal et al. 1998; Lemos \& Rodal 2002; AlcoforadoFilho et al. 2003; Neves 2005; Rodal \& Nascimento 2006), sendo poucos os estudos envolvendo o levantamento de toda a flora vascular (França et al. 1997; Amorim et al. 2005a; CarvalhoSobrinho \& Queiroz 2005; Rodal et al. 2005; Ribeiro-Filho et al. 2009). A importância de levantamentos florísticos mais gerais reside no fato de que um maior conjunto de informações sobre a vegetação amostrada (número de espécies, endemismos, presença de espécies invasoras e etc.) permitiria avaliar o estado de conservação da área que está sendo estudada. Além disso, esses estudos proporcionam a descoberta de novos táxons ou novos registros de ocorrência, contribuindo, portanto, para o conhecimento sobre a diversidade florística e a fitogeografia daquela vegetação.

No estado da Bahia, a grande maioria dos estudos florísticos concentra-se principalmente em áreas de campos rupestres (e.g. Stannard 1995; Zappi et al. 2003; Conceição et al. 2007), formações florestais na Chapada Diamantina (Funch et al. 2005, 2008; Funch 1997, 2008; Stradmann 1997, 2000; Ribeiro-Filho et al. 2009; Couto 2008; Souza 2008), floresta estacional associada a "inselbergues" (França et al. 1997) e na Mata Atlântica (Tavares et al. 1979; Soares-Filho 2000; Sambuichi 2002; Amorim et al. 2005a; Carvalho-Sobrinho \& Queiroz 2005; Neves 2005; Macedo 2007; Martini et al. 2007; Thomas et al. 2009). Pouco se conhece sobre a composição florística dos fragmentos florestais situados na porção centroleste da Bahia, registrando-se até o momento apenas o estudo de Cardoso \& Queiroz (2008), desenvolvido na Serra do Orobó, situada entre os municípios de Ruy Barbosa e Itaberaba, região central do estado.

Este trabalho tem como objetivo principal caracterizar a vegetação de uma floresta semidecídua em Feira de Santana, Bahia, através de sua composição de espécies e classificação fitogeográfica. Especificamente, pretende-se responder as seguintes questões: (i) Qual a composição de espécies desta área de floresta estacional? (ii) Esta composição de espécies é tão diversa quanto a de outras áreas de florestas estacionais do leste do Brasil? (iii) Quais fisionomias de vegetação estão mais relacionadas floristicamente com a área em estudo? 


\section{Material e Métodos}

\section{Área de estudo}

O estudo foi realizado na Serra da Fazenda Retiro (SFR) (entre 12 09'35' S $39^{\circ} 10^{\prime} 40^{\prime \prime} \mathrm{W}$ e $12^{\circ} 10^{\prime} 00^{\prime \prime} \mathrm{S}-39^{\circ} 11^{\prime} 27^{\prime \prime} \mathrm{W}$ ), um remanescente de ca. 13 ha de floresta estacional semidecídua próximo à Rodovia Estrada do Feijão, a ca. 30 km da cidade de Feira de Santana, Bahia (Fig. 1). Este município está localizado na região econômica do Paraguassu, a $110 \mathrm{~km}$ de Salvador, Bahia, inserido na região semiárida. A principal atividade econômica da região é a agricultura, baseada no cultivo de mandioca, feijão e milho, além da criação de bovinos, caprinos e equinos (BAHIA 2006). O desenvolvimento dessas atividades na região, associado ao manejo das pastagens através do fogo, foi responsável por um crescente processo de degradação da vegetação florestal. No entanto, alguns remanescentes de florestas estacionais ainda podem ser encontrados, a exemplo da SFR.

A SFR alcança uma altitude máxima de aproximadamente $500 \mathrm{~m}$. O solo na região é do tipo argissolo vermelho-amarelo eutrófico (BAHIA 2006). Segundo a tipologia climática definida por Thornhwaite (BAHIA 2006), a SFR está sujeita a um clima subúmido a seco (C1d A'), com temperatura média anual de $20^{\circ} \mathrm{C}$ e precipitação média anual de $700 \mathrm{~mm}$.

A unidade de conservação mais próxima da área de estudo é a ARIE Serra do Orobó (Cardoso \& Queiroz 2008), a qual dista ca. $150 \mathrm{~km}$.

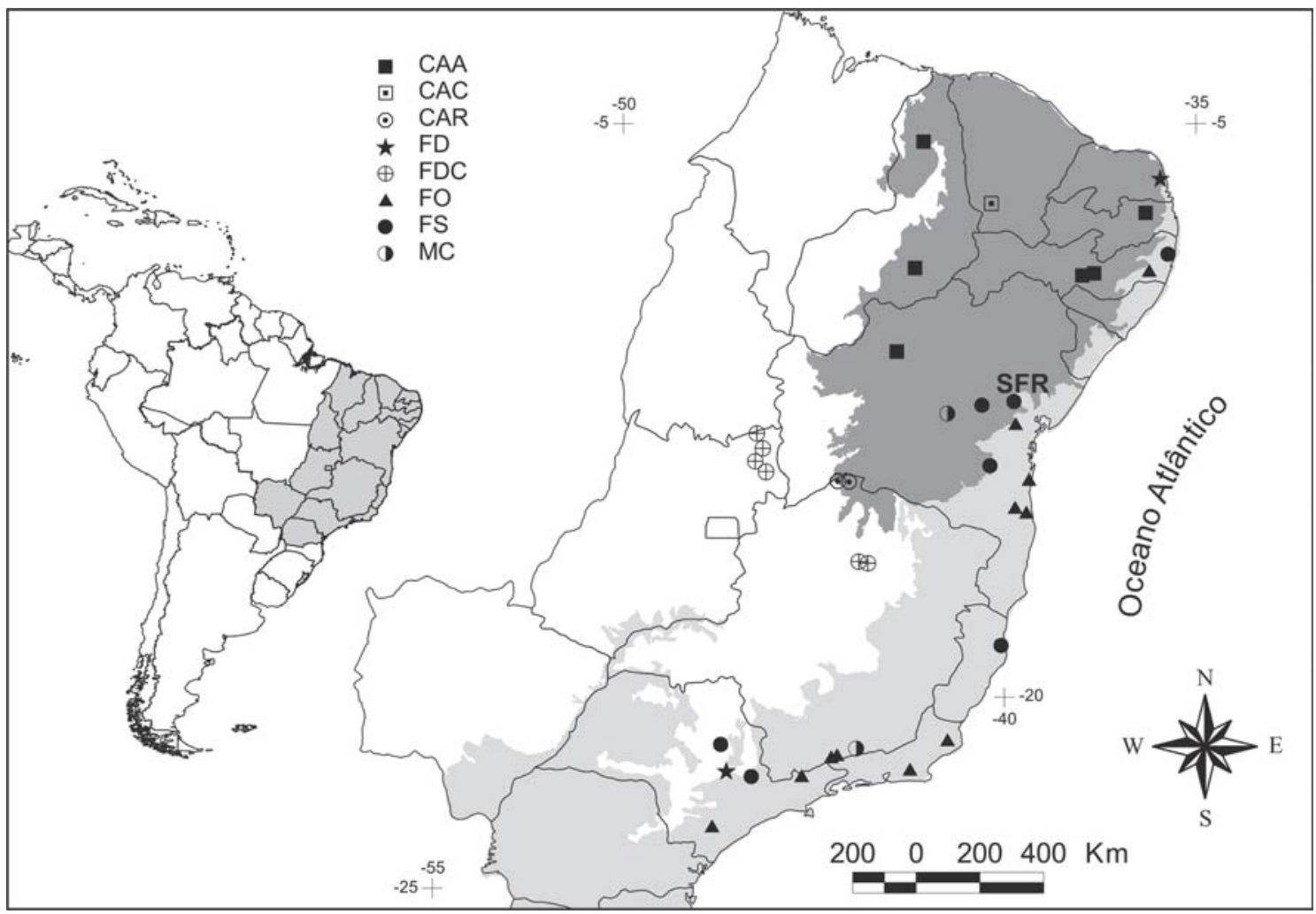

Figura 1 - Localização da Serra da Fazenda Retiro (SFR) no município de Feira de Santana, porção nordeste do estado da Bahia, e das áreas utilizadas na análise de similaridade florística. A porção cinza claro é a delimitação da Mata Atlântica e em cinza escuro a do Bioma Caatinga. As siglas das áreas referem-se às diferentes fisionomias vegetacionais apresentadas na Tabela 1 .

Figure 1 - Location of Serra da Fazenda Retiro (SFR) at the municipality of Feira de Santana, northeastern Bahia, and areas used for comparison in the similarity analysis. The light gray area refers to the delimitation of Atlantic Forest domain and the dark gray area refers to Caatinga Biome. The abbreviations for areas are the distinct vegetation physiognomies showed in Table 1. 


\section{Amostragem florística}

As coletas foram realizadas através de caminhadas aleatórias no período de outubro de 2006 a maio de 2007 e em maio de 2008. Durante esse período, foram feitas nove visitas à região. As plantas foram amostradas em áreas de bordas, clareiras, interior do fragmento florestal e locais sobre afloramento rochoso. Também foram amostradas espécies da caatinga que ocorrem nas partes mais baixas do entorno da serra, além das árvores que restaram nos locais antropizados situados além (até ca. $200 \mathrm{~m}$ dist.) da borda do fragmento. As áreas de bordas foram definidas como o início do fragmento, logo após a transição com a caatinga, enquanto as clareiras seriam as áreas naturais no interior do fragmento. No intuito de complementar a lista florística foram incluídas espécies coletadas, por outros botânicos, em épocas anteriores a 2006, as quais foram depositadas no Herbário da Universidade Estadual de Feira de Santana (HUEFS).

As plantas foram coletadas em estágio reprodutivo (com flor ou fruto), mas eventualmente foram coletados indivíduos estéreis quando o táxon específico era reconhecido em campo. Algumas espécies foram somente listadas, quando se tinha a certeza da identificação das mesmas, devido à dificuldade de acesso para coleta. Todo o material coletado foi herborizado de acordo com os métodos descritos por Mori et al. (1985), e todos os pontos de coleta foram georreferenciados com GPS. Posteriormente, os materiais foram depositados na coleção do HUEFS, e as duplicatas, quando necessário, foram enviadas a especialistas para determinação das espécies. A identificação foi realizada por comparação com os exemplares já depositados no HUEFS, utilização de chaves taxonômicas disponíveis para alguns grupos ou consulta aos especialistas. Neste trabalho, o sistema de classificação adotado para apresentação das famílias foi o proposto pelo APG II (2003).

\section{Análise de similaridade}

Foram selecionadas as espécies arbóreas ou arbustos que potencialmente alcançam mais de $3 \mathrm{~m}$ altura, a partir de um total de 37 áreas, as quais representam diferentes fisionomias vegetacionais do Domínio da Mata Atlântica e do Bioma Caatinga (Tab. 1). Tais fisionomias foram classificadas de acordo com a definição já apresentada nos próprios trabalhos escolhidos para a análise, geralmente seguindo a classificação de Veloso et al. (2001). Em seguida, foi feita uma matriz de presençaausência com as 1.846 espécies selecionadas. As sinonímias e atualização dos nomes das espécies foram checadas em monografias taxonômicas ou na base de dados TROPICOS do Missouri Botanical Garden (http:// www.tropicos.org). Para verificar as relações florísticas entre as áreas, foi feita análise de agrupamento pelo método UPGMA e coeficiente de Jaccard utilizando o programa PAST (Hammer et al. 2001). Neste mesmo programa foi calculado o suporte de "bootstrap" através de 1.000 replicações para verificar a confiabilidade dos agrupamentos.

\section{Resultados}

\section{Caracterização da vegetação}

$\mathrm{Na}$ SFR foi encontrada uma faixa de caatinga arbustiva densa, que ocorre nas áreas degradadas em baixas altitudes ao redor da serra, e um fragmento de floresta estacional semidecídua que se desenvolve a partir dos $250 \mathrm{~m}$ até o topo da serra. Um grande afloramento granítico tipo "inselberg" com ca. $10 \mathrm{~m}$ alt. e ca. $200 \mathrm{~m}$ compr. pode ser encontrado no topo, o qual, quando visto da base da serra, apresenta-se praticamente todo encoberto pela vegetação florestal que o circunda.

No presente estudo, foi dado enfoque apenas ao fragmento florestal, mas foi possível observar que as áreas de caatinga são marcadas pela presença de algumas espécies características desse tipo de vegetação, como Anadenanthera colubrina, Aspidosperma pyrifolium, Cereus jamacaru, Cnidoscolus urens, Mimosa tenuiflora e Senna aversiflora (Prado 2003; Queiroz 2006).

Nos locais mais bem preservados da SFR, principalmente no topo da serra, a vegetação florestal pode ser dividida em diferentes estratos. Dentre as espécies que ocorrem no dossel, cerca de $15 \mathrm{~m}$ alt., destacaram-se Alseis 
Tabela 1 - Relação das áreas utilizadas na análise de similaridade florística considerando as espécies lenhosas acima de $3 \mathrm{~m}$ de altura. Cód. = código de identificação no dendrograma de similaridade (Fig. 2); FO = floresta ombrófila; FS = floresta semidecídua; FD = floresta decídua; FDC = floresta decídua sobre afloramento calcário; $\mathrm{MC}=$ mata ciliar; $\mathrm{CAR}=$ caatinga arbórea; $\mathrm{CAA}=$ caatinga arbustiva sobre solo arenoso; $\mathrm{CAC}=$ caatinga arbustivo-arbórea sobre embasamento cristalino.

Table 1 - List of the areas used in the analysis of floristic similarity considering the woody species larger than $3 \mathrm{~m}$ high. Cód. = abbreviation for areas in the dendrogram of similarity (Fig. 2); FO = moist forest; FS = semideciduous forest; FD $=$ deciduous forest FDC $=$ deciduous forest on limestone outcrop; $\mathrm{MC}=$ gallery forest; $\mathrm{CAR}=$ arboreal caatinga; $\mathrm{CAA}$ $=$ shrubby caatinga on sandy soil; $\mathrm{CAC}=$ tree-shrubby caatinga on crystalline bedrock.

\begin{tabular}{|c|c|c|c|c|c|}
\hline Cód. & Localidade & Estado & Fisionomia & Coordenadas & Referências \\
\hline BAfsa & Feira de Santana & BA & FS & $12^{\circ} 09^{\prime} \mathrm{S}$ e $39^{\circ} 10^{\prime} \mathrm{W}$ & Este estudo \\
\hline BAjeq & Jequié & BA & FS & $13^{\circ} 57^{\prime} \mathrm{S}$ e $40^{\circ} 06^{\prime} \mathrm{W}$ & Macedo (2007) \\
\hline BAcon & Uruçuca/Itacaré & BA & $\mathrm{FO}$ & $14^{\circ} 25^{\prime}$ 'S e $39^{\circ} 06^{\prime} \mathrm{W}$ & Martini et al. (2007) \\
\hline BAoro & Ruy Barbosa & BA & FS & $12^{\circ} 20^{\prime} \mathrm{S}$ e $40^{\circ} 25^{\prime} \mathrm{W}$ & Cardoso \& Queiroz (2008) \\
\hline BAjib & Santa Terezinha & BA & FO & $12^{\circ} 51^{\prime} \mathrm{S}$ e $39^{\circ} 28^{\prime} \mathrm{W}$ & $\begin{array}{l}\text { Neves (2005); Carvalho-Sobrinho \& } \\
\text { Queiroz (2005) }\end{array}$ \\
\hline BAuna & Una & BA & FO & $15^{\circ} 20^{\prime} \mathrm{S}$ e $39^{\circ} 10^{\prime} \mathrm{W}$ & Amorim et al. (2008) \\
\hline BAtei & Jussari & BA & FO & $15^{\circ} 12^{\prime}$ S e $39^{\circ} 29^{\prime} \mathrm{W}$ & Amorim et al. (2005a) \\
\hline BAlen & Lençóis, Rio Lençóis & BA & $\mathrm{MC}$ & $12^{\circ} 33^{\prime} \mathrm{S}$ e $41^{\circ} 24^{\prime} \mathrm{W}$ & Funch (1997) \\
\hline BAibi & $\begin{array}{l}\text { Ibiraba, } \\
\text { Dunas do São Francisco }\end{array}$ & BA & CAA & $10^{\circ} 48^{\prime} \mathrm{S}$ e $42^{\circ} 50^{\prime} \mathrm{W}$ & Rocha et al. (2004) \\
\hline CEaiu & Aiuaba & $\mathrm{CE}$ & $\mathrm{CAC}$ & $06^{\circ} 36^{\prime} \mathrm{S}$ e $40^{\circ} 07^{\prime} \mathrm{W}$ & Lemos (2006) \\
\hline ESlin & Linhares & ES & FS & $19^{\circ} 06^{\prime} \mathrm{S}$ e $39^{\circ} 45^{\prime} \mathrm{W}$ & Rolim et al. (2006) \\
\hline MGjuv & Juvenília & MG & CAR & $14^{\circ} 28^{\prime}$ 'S e $44^{\circ} 11^{\prime} \mathrm{W}$ & Santos et al. (2007) \\
\hline MGmon & Montalvânia & MG & CAR & $14^{\circ} 27^{\prime} \mathrm{S}$ e $44^{\circ} 30^{\prime} \mathrm{W}$ & Santos et al. (2007) \\
\hline MGmcl & Montes Claros & MG & FDC & $16^{\circ} 44^{\prime} \mathrm{S}$ e $43^{\circ} 54^{\prime} \mathrm{W}$ & Santos et al. (2007) \\
\hline MGjur & Juramento & MG & FDC & $16^{\circ} 46^{\prime} \mathrm{S}$ e $43^{\circ} 39^{\prime} \mathrm{W}$ & Santos et al. (2007) \\
\hline MGbho & Belo Horizonte & $\mathrm{MG}$ & $\mathrm{MC}$ & $22^{\circ} 01^{\prime} \mathrm{S}$ e $43^{\circ} 59^{\prime} \mathrm{W}$ & Meyer et al. (2004) \\
\hline MGboc & Bocaina de Minas & MG & FO & $22^{\circ} 13^{\prime} \mathrm{S}$ e $44^{\circ} 32^{\prime} \mathrm{W}$ & Carvalho et al. (2005) \\
\hline GOiac & Iaciara & GO & FDC & $14^{\circ} 03^{\prime}$ 'S e $46^{\circ} 29^{\prime} \mathrm{W}$ & Felfili et al. (2007) \\
\hline GOcan & $\begin{array}{l}\text { São Domingos, } \\
\text { Faz. Canadá }\end{array}$ & $\mathrm{GO}$ & FDC & $13^{\circ} 04^{\prime} \mathrm{S}$ e $46^{\circ} 44^{\prime} \mathrm{W}$ & Silva \& Scariot (2004a) \\
\hline GOsvi & $\begin{array}{l}\text { São Domingos, } \\
\text { Faz. São Vicente }\end{array}$ & GO & FDC & $13^{\circ} 31^{\prime} \mathrm{S}$ e $46^{\circ} 29^{\prime} \mathrm{W}$ & Silva \& Scariot (2004b) \\
\hline GOsjo & São Domingos, Faz. São José & $\mathrm{GO}$ & FDC & $13^{\circ} 49^{\prime} \mathrm{S}$ e $46^{\circ} 41^{\prime} \mathrm{W}$ & Silva \& Scariot (2003) \\
\hline PBare & $\begin{array}{l}\text { Areia e Remígio, } \\
\text { Agreste Paraibano }\end{array}$ & PB & CAA & $06^{\circ} 52^{\prime} \mathrm{S}$ e $35^{\circ} 47^{\prime} \mathrm{W}$ & Pereira et al. (2002) \\
\hline PEbui & Buíque & PE & CAA & $08^{\circ} 35^{\prime} \mathrm{S}$ e $37^{\circ} 15^{\prime} \mathrm{W}$ & Gomes et al. (2006) \\
\hline PEbon & Bonito & PE & FO & $08^{\circ} 30^{\prime} \mathrm{S}$ e $35^{\circ} 42^{\prime} \mathrm{W}$ & Rodal et al. (2005) \\
\hline PEslo & São Lourenço da Mata & $\mathrm{PE}$ & FS & $08^{\circ} 03^{\prime}$ S e $35^{\circ} 09^{\prime} \mathrm{W}$ & Andrade \& Rodal (2004) \\
\hline PEibi & $\begin{array}{l}\text { Ibimirim, } \\
\text { Bacia Tucano-Jatobá }\end{array}$ & $\mathrm{PE}$ & CAA & $08^{\circ} 39^{\prime} \mathrm{S}$ e $37^{\circ} 35^{\prime} \mathrm{W}$ & Rodal et al. (1999) \\
\hline PIcap & $\begin{array}{l}\text { Parque Nacional } \\
\text { Serra da Capivara }\end{array}$ & PI & CAA & $08^{\circ} 26^{\prime} \mathrm{S}$ e $42^{\circ} 19^{\prime} \mathrm{W}$ & $\begin{array}{l}\text { Lemos \& Rodal (2002); } \\
\text { Lemos (2004) }\end{array}$ \\
\hline PIcma & Complexo de Campo Maior & PI & CAA & $04^{\circ} 51^{\prime}$ 'S e $42^{\circ} 04^{\prime} \mathrm{W}$ & Farias \& Castro (2004) \\
\hline RNmac & Macaíba & $\mathrm{RN}$ & FD & $05^{\circ} 53^{\prime} \mathrm{S}$ e $35^{\circ} 23^{\prime} \mathrm{W}$ & Cestaro \& Soares (2004) \\
\hline RJita & Itatiaia & $\mathrm{RJ} / \mathrm{MG}$ & $\mathrm{FO}$ & $22^{\circ} 15^{\prime} \mathrm{S}$ e $44^{\circ} 35^{\prime} \mathrm{W}$ & Pereira et al. (2006) \\
\hline RJgoy & Campos dos Goytacazes & RJ & FO & $21^{\circ} 48^{\prime} \mathrm{S}$ e $14^{\circ} 40^{\prime} \mathrm{W}$ & Moreno et al. (2003) \\
\hline RJsja & Silva Jardim & RJ & FO & $22^{\circ} 37^{\prime}$ S e $42^{\circ} 28^{\prime} \mathrm{W}$ & Carvalho et al. (2006) \\
\hline SPpir & Piracicaba & SP & FD & $22^{\circ} 39^{\prime} \mathrm{S}$ e $47^{\circ} 39^{\prime} \mathrm{W}$ & Ivanauskas \& Rodrigues (2000) \\
\hline SPcam & Campinas & SP & FS & $22^{\circ} 50^{\prime}$ S e $46^{\circ} 56^{\prime} \mathrm{W}$ & Santos \& Kinoshita (2003) \\
\hline SPsca & São Carlos & SP & FS & $21^{\circ} 55^{\prime} \mathrm{S}$ e $47^{\circ} 48^{\prime} \mathrm{W}$ & Silva \& Soares (2003) \\
\hline SPpin & Pindamonhangaba & SP & FO & $22^{\circ} 48^{\prime} \mathrm{S}$ e $45^{\circ} 32^{\prime} \mathrm{W}$ & Gomes et al. (2005) \\
\hline SPsba & Sete Barras & SP & FO & $24^{\circ} 14^{\prime}$ 'S e $48^{\circ} 04^{\prime} \mathrm{W}$ & Guilherme et al. (2004) \\
\hline
\end{tabular}


floribunda, Aspidosperma parvifolium, A. polyneuron, Byrsonima nitidifolia, Cavanillesia arborea, Cnidoscolus oligandrus, Gallesia integrifolia, Guazuma ulmifolia, Ficus nymphaeifolia, Peltophorum dubium e Pseudopiptadenia bahiana. Ocorreram também árvores emergentes de Goniorrhachis marginata com $25 \mathrm{~m}$ de altura.

No sub-bosque, as espécies arbóreas mais comuns foram Casearia sylvestris, Brasiliopuntia brasiliensis, Clusia nemorosa, C. melchiorii, Jacaratia spinosa, Machaerium acutifolium, Maprounea guianensis, Psidium cauliflorum, Handroanthus impetiginosus e Zollernia ilicifolia. Dentre as espécies arbustivas, Acalypha amblyodonta, Actinostemon appendiculatus, Capparis frondosa, Eugenia candoleana, Faramea hyacinthina, Guapira hirsuta, Helicteres macropetala, Sapium glandulosum, Solanum depauperatum, Syphoneugena sp. e Urera baccifera. As espécies herbáceas mais comuns no interior do sub-bosque foram Aechmea multiflora, Billbergia fosteriana, Dichorisandra hexandra, Hybanthus verrucosus, Justicia humuliflora, Microtea bahiensis, Pseuderanthemum modestum, Raddia portoi e Wissadula amplissima. As espécies mais comuns sobre os afloramentos rochosos foram Begonia saxicola, Calathea sp., Epidendrum anceps, Hohenbergia stellata, Maranta protracta, Neomarica sp. nov., Sinningia barbata e Tibouchina lithophila.

As epífitas foram representadas por Anthurium pentaphyllum, Cattleya aclandiae, Epiphyllum phyllanthus, Oncidium barbatum, Rhipsalis lindbergiana e Tillandsia recurvata.

Devido ao histórico recente de degradação do fragmento florestal pelo fogo, algumas áreas não apresentaram a vegetação estratificada verticalmente. As espécies mais comuns nessas áreas e nas clareiras foram Adenocalymma comosum, Aspilia hispidantha, Cordia trichotoma, Crotalaria holosericea, Fevillea trilobata, Maclura tinctoria, Mimosa tenuiflora, Priva bahiensis e Trichilia hirta. A lista completa das espécies coletadas em SFR pode ser visualizada na Tabela 2 .

\section{Diversidade florística}

Foram coletadas na SFR 197 espécies incluídas em 160 gêneros e 61 famílias botânicas (Tab. 2). Deste total 90\% (177 spp.) foram identificadas em nível específico, $9 \%$ (18 spp.) em nível genérico e apenas 1\% (2 spp.) permaneceram em família. Excluindo as espécies que foram coletadas na caatinga do entorno da serra, na fisionomia florestal e no afloramento associado foram coletadas 173 espécies, distribuídas em 143 gêneros e 59 famílias. Nestes últimos ambientes, dentre as espécies amostradas, 81 (47\%) são árvores, $18(10 \%)$ arbustos, $58(34 \%)$ ervas terrestres ou epífitas e $15(9 \%)$ trepadeiras herbáceas ou lenhosas. As famílias a seguir citadas representaram $35 \%$ da flora vascular da floresta semidecídua da SFR: Myrtaceae (15 spp.), Euphorbiaceae (13), Leguminosae (12), Malvaceae (7), Orchidaceae (7) e Rubiaceae (7). Dentre as demais famílias, 25 foram representadas apenas por uma espécie cada.

A riqueza de espécies por gênero na flora do SFR foi muito baixa, considerando que 134 gêneros $(83,75 \%)$ amostrados contribuíram com apenas uma espécie cada. Somente os gêneros Eugenia, Capparis, Aspidosperma, Erythroxylon, Casearia e Cordia apresentaram mais que três espécies, sendo Eugenia o mais diverso (7 spp.).

Foi registrada a ocorrência de três possíveis espécies novas para a ciência, pertencentes aos seguintes gêneros: Neomarica-Iridaceae (A.Gil, com. pess.), Pseudobombax - Malvaceae (J.G.Carvalho-Sobrinho, com. pess.) e Solanum - Solanaceae (D.Cardoso, obs. pess.). Dentre as espécies coletadas, Psidium cauliflorum (Myrtaceae) era conhecida apenas pelo material tipo e Epidendrum anceps (Orchidaceae) havia sido registrada somente na Floresta Atlântica do sul da Bahia (C.van den Berg, com. pess.).

\section{Similaridade florística}

Na análise de similaridade (Fig. 2) foi possível observar a formação de dois grandes grupos: (i) o primeiro formado com $52 \%$ de suporte de "bootstrap" (SB), incluindo todas as áreas de 
Tabela 2 - Lista de espécies coletadas na Serra da Fazenda Retiro (SFR), município de Feira de Santana, Bahia. As espécies marcadas em asterisco $\left(^{*}\right)$ e por um círculo aberto $\left(^{\circ}\right)$ foram encontradas, respectivamente, nos levantamentos florísticos de Amorim et al. (2005a) e Cardoso \& Queiroz (2008) em áreas de Mata Atlântica s.l. Hábito: Erv = ervas terrestres ou epífitas; Tre = trepadeira herbácea ou lenhosa; Arb = arbusto; Arv = árvore. Coletores: DC = D.Cardoso; JN = J.S.Novais; LQ = L.P.de Queiroz; MF = M.H.S.Ferreira; RO = R.P.de Oliveira. Habitat: FSE = floresta semidecídua; $\mathrm{AFL}=$ afloramento rochoso; $\mathrm{CAA}=$ caatinga.

Table 2 - Species list of the plants found in the Serra da Fazenda Retiro (SFR), at municipality of Feira de Santana, Bahia. The asterisk $\left(^{*}\right)$ and the circle $\left({ }^{\circ}\right)$ after the species names highlights the species found in the floristic surveys of Atlantic Forest s.l. by Amorim et al. (2005a) and by Cardoso \& Queiroz (2008), respectively. Abbreviations for habit: Erv = terrestrial herb or epiphyte; Tre = herbaceous or woody vine; Arb = shrub; Arv = tree. Abbreviations for collectors: DC = D.Cardoso; JN = J.S.Novais; LQ = L.P.de Queiroz; MF = M.H.S.Ferreira; RO = R.P.de Oliveira. Abbreviations for habitat: FSE = semideciduous forest; $\mathrm{AFL}=$ rocky outcrop; $\mathrm{CAA}=$ caatinga.

\begin{tabular}{|c|c|c|c|c|}
\hline Família & Espécie & Hábito & Vouchers & Habitat \\
\hline \multirow[t]{5}{*}{ ACANTHACEAE } & Dicliptera mucronifolia Nees & Erv & LQ1029 & FSE \\
\hline & Schaueria humuliflora Nees & Erv & DC 1480; MF 20, 21 & FSE \\
\hline & $\begin{array}{l}\text { Pseuderanthemum modestum } \\
\text { (Nees) Radlk. }\end{array}$ & Erv & DC 1430, 1907; JN 22 & FSE \\
\hline & Ruellia cearensis Lindau* & Erv & DC 1445,$1396 ; \mathrm{JN} 49$ & FSE \\
\hline & Ruellia curviflora Nees \& Mart.* & Erv & LQ1013 & FSE \\
\hline AGAVACEAE & Herreria salsaparilha Mart. $^{\circ}$ & Tre & DC 1425 & FSE \\
\hline AMARYLLIDACEAE & Bomarea rosea (Ruiz \& Pav.) Herb. & Tre & DC 1395; JN 32 & FSE \\
\hline \multirow[t]{3}{*}{ ANACARDIACEAE } & Myracrodruon urundeuva Allemão & Arv & DC 1966 & CAA \\
\hline & Spondias venulosa Mart. ex Benth. & Arv & DC 1935 & FSE \\
\hline & Schinopsis brasiliensis Engl. & Arv & RO 1537 & CAA \\
\hline \multirow[t]{3}{*}{ APOCYNACEAE } & Aspidosperma parvifolium A.DC.*o & Arv & DC 1475,1928 & FSE \\
\hline & Aspidosperma polyneuron Müll.Arg. & Arv & DC 1916 & FSE \\
\hline & Aspidosperma pyrifolium Mart. $^{\circ}$ & Arv & MF 22 & CAA \\
\hline \multirow[t]{5}{*}{ ARACEAE } & Anthurium affine Schott & Erv & DC 1438 & FSE \\
\hline & Anthurium pentaphyllum (Aubl.) G.Don*o & Tre & DC 1446; MF 29 & FSE \\
\hline & Philodendron acutatum Schott & Erv & DC 1900 & FSE \\
\hline & Spathicarpa gardneri Schott & Erv & RO 1544 & FSE \\
\hline & Xanthosoma helleborifolium (Jacq.) Schott & Erv & DC 1945 & FSE \\
\hline ARALIACEAE & Aralia warmingiana (Marchal) J.Wen & Arv & DC 1962 & FSE \\
\hline ARECACEAE & Syagrus coronata Becc. ${ }^{\circ}$ & Arv & Não coletado & CAA \\
\hline \multirow[t]{4}{*}{ ASTERACEAE } & Aspilia hispidantha H.Rob. & Erv & JN 15 & FSE \\
\hline & Bidens subalternans DC. & Erv & LQ 1028 & FSE \\
\hline & Delilea biflora $\mathrm{Kuntze}^{\circ}$ & Erv & JN35 & FSE \\
\hline & Trixis antimenorrhoea (Schrank) Kuntze & Erv & LQ1379 & CAA \\
\hline \multirow[t]{5}{*}{ BIGNONIACEAE } & Adenocalymma comosum (Cham.) A.DC. & Tre & $\mathrm{JN} 12$ & FSE \\
\hline & $\begin{array}{l}\text { Clytostoma convolvuloides } \\
\text { Bureau \& K.Schum. }\end{array}$ & Tre & LQ1015 & FSE \\
\hline & $\begin{array}{l}\text { Handroanthus chrysotrichus } \\
\text { (Mart. ex A.DC.) Mattos }\end{array}$ & Arv & DC 1904 & FSE \\
\hline & $\begin{array}{l}\text { Handroanthus impetiginosus } \\
\text { (Mart. ex DC.) Mattos }\end{array}$ & Arv & DC 1909 & FSE \\
\hline & Tabebuia roseo-alba (Ridl.) Sandwith* & Arv & DC 1915 & FSE \\
\hline
\end{tabular}




\begin{tabular}{|c|c|c|c|c|}
\hline Família & Espécie & Hábito & Vouchers & Habitat \\
\hline \multirow[t]{2}{*}{ BEGONIACEAE } & Begonia reniformis Dryander & Erv & DC 1403 & AFL \\
\hline & Begonia saxicola A.DC. & Erv & DC 1409 & AFL \\
\hline \multirow[t]{3}{*}{ BORAGINACEAE } & Cordia globosa & Arb & LQ 1037 & CAA \\
\hline & Cordia superba*o & Arb/Arv & LQ 1042 & FSE \\
\hline & Cordia trichotoma (Vell.) Arrab. ex Steud. & Arv & DC 1951 & FSE \\
\hline \multirow[t]{4}{*}{ BROMELIACEAE } & Aechmea multiflora L.B.Sm. & Erv & DC 1432 & FSE \\
\hline & Billbergia fosteriana L.B.Sm. & Erv & DC 1946 & AFL \\
\hline & Hohenbergia stellata Schult. f. & Erv & DC 1467 & AFL \\
\hline & Tillandsia recurvata $\mathrm{L}$. & Erv & DC 1990 & FSE \\
\hline \multirow[t]{6}{*}{ CACTACEAE } & $\begin{array}{l}\text { Brasiliopuntia brasiliensis } \\
\text { (Willd.) A.Berger*o }\end{array}$ & Arv & $\begin{array}{l}\text { DC 1939; MF 24; } \\
\text { JN } 54\end{array}$ & FSE \\
\hline & Cereus jamacaru DC. & Arv & Não coletado & CAA \\
\hline & Epiphyllum phyllanthus (L.) Haw.*o & Erv & DC 1442 & FSE \\
\hline & $\begin{array}{l}\text { Pilosocereus pentaedrophorus } \\
\text { (J.F.Cels) Byles \& G.D.Rowley }\end{array}$ & Arb/Arv & DC 1991 & FSE \\
\hline & $\begin{array}{l}\text { Pseudoacanthocereus brasiliensis } \\
\text { (Britton \& Rose) F.Ritter }\end{array}$ & Erv & DC 1451 & FSE \\
\hline & Rhipsalis lindbergiana K.Schum. & Erv & DC 1437 & FSE \\
\hline CAMPANULACEAE & Lobelia xalapensis Kunth & Erv & DC 1456 & FSE \\
\hline CANNABACEAE & Celtis iguanaea (Jacq.) Sarg. & Arv & DC 1932 & FSE \\
\hline CARICACEAE & Jacaratia spinosa (Aubl.) A.DC. & Arv & DC 1949 & FSE \\
\hline \multirow[t]{2}{*}{ CELASTRACEAE } & Maytenus distichophylla Mart. ex Reiss. & Arv & DC 1975 & FSE \\
\hline & Maytenus quadrangulata (Schrad.) Loes. ${ }^{\circ}$ & Arv & DC 1934 & FSE \\
\hline \multirow[t]{2}{*}{ CLUSIACEAE } & Clusia nemorosa G.Mey. $^{\circ}$ & Arv & DC 1449 & FSE \\
\hline & Clusia melchiorii Gleason & Arv & DC 1440,1953 & FSE \\
\hline \multirow{4}{*}{ COMMELINACEAE } & Aneilema brasiliense C.B.Clarke & Erv & DC1398 & FSE \\
\hline & Commelina benghalensis L. & Erv & JN27 & FSE \\
\hline & $\begin{array}{l}\text { Dichorisandra glabrescens } \\
\text { Aona \& Amaral }\end{array}$ & Erv & DC1481 & FSE \\
\hline & Dichorisandra hexandra (Aubl.) Standl.* & Erv & DC1891, 1896 & FSE \\
\hline CONVOLVULACEAE & Ipomoea sp. & Tre & JN 11 & FSE \\
\hline \multirow[t]{5}{*}{ CRUCIFERAE } & Capparis brasiliana $\mathrm{DC} .^{\circ}$ & Arb/Arv & DC 1479 & FSE \\
\hline & Capparis flexuosa (L.) L. ${ }^{\circ}$ & Arb & DC 1933 & CAA \\
\hline & Capparis frondosa Jacq.* & Arb/Arv & DC 1920, 1986 & FSE \\
\hline & Capparis yco Mart. & Arb & DC 1927 & CAA \\
\hline & Cleome sp. & Erv & DC 1405; JN 30 & FSE \\
\hline \multirow[t]{2}{*}{ CUCURBITACEAE } & Cucurbitaceae & Tre & DC 1427 & FSE \\
\hline & Fevillea trilobata L.* & Tre & $\begin{array}{l}\text { DC } 1427,1428 \\
1433 ; \text { JN } 58\end{array}$ & FSE \\
\hline \multirow[t]{2}{*}{ CYPERACEAE } & Cyperaceae & Erv & DC 1905 & FSE \\
\hline & Cyperus simplex Kunth & Erv & DC 1988 & FSE \\
\hline DIOSCOREACEAE & Dioscorea sp. & Tre & DC 1989 & FSE \\
\hline \multirow[t]{3}{*}{ ERYTHROXYLACEAE } & Erythroxylum citrifolium A.St.-Hil. & Arb/Arv & DC 1980 & FSE \\
\hline & Erythroxylum petrae-cabralli Plowman* & Arb/Arv & DC 1901 & FSE \\
\hline & Erythroxylum sp. & Arb & DC 1464; JN 56 & FSE \\
\hline EUPHORBIACEAE & $\begin{array}{l}\text { Acalypha amblyodonta } \\
\text { (Müll.Arg.) Müll.Arg. }^{\circ}\end{array}$ & Arb & DC 1422; JN 60 & FSE \\
\hline
\end{tabular}




\begin{tabular}{|c|c|c|c|c|}
\hline Família & Espécie & Hábito & Vouchers & Habitat \\
\hline & Acalypha brasiliensis Müll.Arg.*o & Arb & JN 14 & FSE \\
\hline & Actinostemon appendiculatus Jabl.* & Arb/Arv & $\begin{array}{l}\text { DC 1441, 1937; } \\
\text { JN } 47,55\end{array}$ & FSE \\
\hline & $\begin{array}{l}\text { Actinostemon concolor } \\
\text { (Spreng.) Müll.Arg. }{ }^{\circ}\end{array}$ & Arb/Arv & DC 1913 & FSE \\
\hline & Astraea lobata (L.) Klotzsch ${ }^{\circ}$ & Arb & JN 24 & FSE \\
\hline & Bernardia tamanduana (Baill.) Müll.Arg. & Arb & LQ1007 & FSE \\
\hline & $\begin{array}{l}\text { Cnidoscolus oligandrus } \\
\text { (Müll.Arg.) Pax*० }\end{array}$ & Arv & JN 59 & FSE \\
\hline & Cnidoscolus urens (L.) Arthur ${ }^{\circ}$ & Arb & JN29 & CAA \\
\hline & Dalechampia brasiliensis Lam. ${ }^{\circ}$ & Tre & JN 16 & FSE \\
\hline & Maprounea guianensis Aubl. ${ }^{\circ}$ & Arb/Arv & MF 23 & FSE \\
\hline & Phylyra brasiliensis $\mathrm{Klotzsch}^{\circ}$ & Arb/Arv & DC 1944 & FSE \\
\hline & Sapium glandulosum (L.) Morong & Arb/Arv & DC 1895 & FSE \\
\hline & Savia sessiliflora (Sw.) Willd. & Arb & DC 1436, 1964 & FSE \\
\hline & Sebastiania brasiliensis Spreng.* & Arv & DC 1898 & FSE \\
\hline & Tragia lessertiana (Baill.) Müll.Arg. & Arb & LQ1380 & CAA \\
\hline GESNERIACEAE & Sinningia barbata Nichols.*o & Erv & DC 1404,1452 & AFL \\
\hline \multirow[t]{6}{*}{ GRAMINAE } & Ichnanthus sp. & Erv & DC 1893 & FSE \\
\hline & Lasiacis ligulata Hitchc. \& Chase & Erv & LQ1383 & FSE \\
\hline & Panicum trichoides Sw. & Erv & JN 25 & FSE \\
\hline & Panicum venezuelae Hack. & Erv & DC 1987 & FSE \\
\hline & Raddia portoi Kuhlm. ${ }^{\circ}$ & Erv & DC 1936 & FSE \\
\hline & Setaria sp. & Erv & RO 1540 & CAA \\
\hline IRIDACEAE & Neomarica sp. nov. & Erv & DC 1426 & AFL \\
\hline LAMIACEAE & Ocimum campechianum Mill. $^{\circ}$ & Erv & JN 48; LQ 1034 & FSE \\
\hline \multirow{6}{*}{$\begin{array}{l}\text { LEGUMINUSAE } \\
\text { Caesalpinioideae }\end{array}$} & & & & \\
\hline & Bauhinia cheilantha (Bong.) Steud. & Arb & DC1974 & CAA \\
\hline & Goniorrhachis marginata Taub.*o & Arv & DC 1929, 1938 & FSE \\
\hline & Peltophorum dubium (Spreng.) Taub. ${ }^{\circ}$ & Arv & DC 1912 & FSE \\
\hline & $\begin{array}{l}\text { Poincianella pyramidalis } \\
\text { (Tul.) L.P.Queiroz }\end{array}$ & Arb & DC 1930 & CAA \\
\hline & $\begin{array}{l}\text { Senna aversiflora } \\
\text { (Hebert.) H.S.Irwin \& Barneby }\end{array}$ & Arb & DC 1391 & CAA \\
\hline \multirow[t]{7}{*}{ Mimosoideae } & Anadenanthera colubrina (Vell.) Brenan ${ }^{\circ}$ & Arv & DC 2256 & CAA \\
\hline & Chloroleucon dumosum (Benth.) G.P.Lewis & Arv & DC 1921 & FSE \\
\hline & Mimosa tenuiflora (Willd.) Poir. & Arv & Não coletado & CAA \\
\hline & $\begin{array}{l}\text { Parapiptadenia blanchetti } \\
\text { (Benth.) Vaz \& M.P.Lima }\end{array}$ & Arv & DC 1918, 1943 & FSE \\
\hline & $\begin{array}{l}\text { Pseudopiptadenia bahiana } \\
\text { G.P.Lewis \& M.P.Lima*o }\end{array}$ & Arv & DC 1942, 1969 & FSE \\
\hline & $\begin{array}{l}\text { Senegalia bahiensis } \\
\text { (Benth.) Seigler \& Ebinger }\end{array}$ & Arv & DC 1956 & CAA \\
\hline & Senegalia sp. & Arb & DC 1950 & CAA \\
\hline \multirow[t]{2}{*}{ Papilionoideae } & Canavalia parviflora Benth. & Tre & DC 1888 & FSE \\
\hline & Crotalaria holosericea Nees \& Mart. & Arb & DC 1454 & FSE \\
\hline
\end{tabular}




\begin{tabular}{|c|c|c|c|c|}
\hline Família & Espécie & Hábito & Vouchers & Habitat \\
\hline & Dioclea violacea Mart. ex Benth. $^{\circ}$ & Tre & DC 1940 & FSE \\
\hline & Erythrina velutina Willd. & Arv & DC 1952 & CAA \\
\hline & Lonchocarpus sericeus (Poir.) DC. & Arv & DC 1976 & FSE \\
\hline & Machaerium acutifolium Vogel & Arv & DC 1474 & FSE \\
\hline & 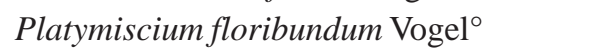 & Arv & DC 1911 & FSE \\
\hline & Zollernia ilicifolia (Brongn.) Vogel ${ }^{\circ}$ & Arv & DC 2255 & FSE \\
\hline LYTHRACEAE & Cuphea glutinosa Cham. \& Schlecht. & Erv & DC 1459 & FSE \\
\hline \multirow[t]{2}{*}{ MALPIGHIACEAE } & Byrsonima nitidifolia A.Juss. ex Char. & Arv & DC 1477 & FSE \\
\hline & Dicella bracteosa (A.Juss.) Griseb.*o & Tre & Não coletado & FSE \\
\hline \multirow[t]{10}{*}{ MALVACEAE } & Abutilon pauciflorum A.St.-Hil. & Arb & DC 1471 & CAA \\
\hline & Abutilon scabridum K.Schum. & Arb & LQ 1032 & CAA \\
\hline & Cavanillesia arborea K.Schum.*॰ & Arv & DC1981;LQ 1035 & FSE \\
\hline & Ceiba glaziovii (Kuntze) K.Schum. & Arv & DC 1957 & FSE \\
\hline & Guazuma ulmifolia Lam.*o & Arv & DC 1388, 1439; JN 13 & FSE \\
\hline & Helicteres macropetala A.St.-Hil. ${ }^{\circ}$ & Arb/Arv & DC 1397; JN 18 & FSE \\
\hline & Melochia tomentosa $\mathrm{L}$. & Arb & LQ1039 & CAA \\
\hline & $\begin{array}{l}\text { Pseudobombax grandiflorum } \\
\text { (Cav.) A.Robyns }\end{array}$ & Arv & DC 1897 & FSE \\
\hline & Pseudobombax sp. nov. & Arv & RO 1538 & FSE \\
\hline & Wissadula amplissima (L.) R.E.Fr. $^{\circ}$ & Erv & DC 1458 & FSE \\
\hline \multirow{2}{*}{ MARANTACEAE } & Calathea sp. & Erv & JN 53 & AFL \\
\hline & Maranta protracta Miq. & Erv & DC 1424,1457 & AFL \\
\hline MELASTOMATACEAE & Tibouchina lithophila Wurdack & Arb/Arv & DC 1466 & AFL \\
\hline MELIACEAE & Trichilia hirta $\mathrm{L} .{ }^{\circ}$ & Arv & RO 1541 & FSE \\
\hline \multirow[t]{3}{*}{ MORACEAE } & Ficus citrifolia Mill. $^{\circ}$ & Arv & DC 1902 & FSE \\
\hline & Ficus nymphaeifolia Mill.*o & Arv & DC 1906, 1985 & FSE \\
\hline & Maclura tinctoria (L.) D.Don ex Steud.*o & Arv & DC 1947 & FSE \\
\hline \multirow[t]{15}{*}{ MYRTACEAE } & Calyptranthes sp. & Arb & DC 1470 & FSE \\
\hline & $\begin{array}{l}\text { Campomanesia dichotoma } \\
\text { (O.Berg) Mattos }\end{array}$ & Arb/Arv & DC 1958 & FSE \\
\hline & Campomanesia viatoris Landrum $^{\circ}$ & Arb/Arv & DC 1972 & FSE \\
\hline & Eugenia candoleana DC.* & Arb/Arv & DC 1948 & FSE \\
\hline & Eugenia florida $\mathrm{DC} .^{\circ}$ & Arb/Arv & DC 1892, 1973 & FSE \\
\hline & Eugenia hyemalis Cambess. & Arb/Arv & DC 1959 & FSE \\
\hline & Eugenia sp. 1 & Arb & DC 1453,1447 & FSE \\
\hline & Eugenia sp. 2 & Arb & MF 28 & FSE \\
\hline & Eugenia sp. 3 & Arb & MF 28 & FSE \\
\hline & Eugenia sp. 4 & Arb & DC 1478 & FSE \\
\hline & Myrcia sp. 1 & Arb & DC 1890 & FSE \\
\hline & Myrcia sp. 2 & Arv & DC 1399 & FSE \\
\hline & Plinia cauliflora (DC.) Kausel & Arv & DC 1970 & FSE \\
\hline & Psidium cauliflorum Landrum \& Sobral & Arv & $\begin{array}{l}\text { DC } 1435,1469,1968 ; \\
\text { MF } 27\end{array}$ & FSE \\
\hline & Syphoneugena sp. & Arb/Arv & DC 1919, 1983 & FSE \\
\hline \multirow[t]{2}{*}{ NYCTAGINACEAE } & Guapira opposita (Vell.) Reitz*o & Arv & DC 1978 & FSE \\
\hline & Guapira hirsuta (Choisy) Lundell ${ }^{\circ}$ & Arb/Arv & DC 1979 & FSE \\
\hline OPILIACEAE & Agonandra excelsa Griseb. & Arv & DC 1971 & FSE \\
\hline
\end{tabular}




\begin{tabular}{|c|c|c|c|c|}
\hline Família & Espécie & Hábito & Vouchers & Habitat \\
\hline \multirow[t]{7}{*}{ ORCHIDACEAE } & $\begin{array}{l}\text { Acianthera saundersiana (Rchb.f.) } \\
\text { Pridgeon \& M.W.Chase }\end{array}$ & Erv & DC 1465 & FSE \\
\hline & Brassavola tuberculata Hook. & Erv & JN 20 & FSE \\
\hline & Cattleya aclandiae Lindl. & Erv & DC 1443; LQ 1406 & FSE \\
\hline & Epidendrum anceps Jacq. & Erv & DC 1461 & AFL \\
\hline & Notylia sp. & Erv & JN 51 & FSE \\
\hline & Oncidium barbatum Lindl.* & Erv & DC 1992; MF 26 & FSE \\
\hline & $\begin{array}{l}\text { Trichocentrum pumilum (Lindl.) } \\
\text { M.W.Chase \& N.H.Williams }\end{array}$ & Erv & DC 1472 & FSE \\
\hline OXALIDACEAE & Oxalis alstonii Lourteig & Erv & $\begin{array}{l}\text { DC 1406; JN 23; } \\
\text { MF } 30\end{array}$ & FSE \\
\hline \multirow[t]{2}{*}{ PHYLLANTACEAE } & $\begin{array}{l}\text { Astrocasia jacobinensis } \\
\text { (Müll.Arg.) G.L.Webster }\end{array}$ & Arb/Arv & DC 1925; LQ 1386 & FSE \\
\hline & Phyllanthus niruri L.$^{\circ}$ & Erv & DC 1455 & FSE \\
\hline \multirow[t]{4}{*}{ PHYTOLACCACEAE } & Gallesia integrifolia (Spreng.) Harms*॰ & Arv & DC 1917 & FSE \\
\hline & Petiveria alliacea L.* & Erv & JN 19, 50 & FSE \\
\hline & $\begin{array}{l}\text { Microtea bahiensis } \\
\text { M.S.Marchioretto \& J.C.de Siqueira }\end{array}$ & Erv & DC 1448 & FSE \\
\hline & Rivina humilis L.*o & Arb & LQ1036 & FSE \\
\hline PIPERACEAE & Peperomia trineura Miq. & Erv & DC 1400 & FSE \\
\hline POLYGONACEAE & Coccoloba declinata (Vell.) Mart.*o & Arb/Arv & DC 1963, 1977 & FSE \\
\hline PORTULACACEAE & Talinum triangulare (Jacq.) Willd. ${ }^{\circ}$ & Erv & JN 21; LQ 1033 & FSE \\
\hline \multirow[t]{7}{*}{ RUBIACEAE } & Alseis floribunda Schott*o & Arv & DC 1961; LQ 1003 & FSE \\
\hline & Coutarea hexandra (Jacq.) K.Schum. ${ }^{\circ}$ & Arb/Arv & 1899 & FSE \\
\hline & Faramea hyacinthina Mart.*० & Arb/Arv & $\begin{array}{l}\text { DC 1394, 1468, } \\
1941 ; \text { JN } 57\end{array}$ & FSE \\
\hline & Leptoscela ruellioides Hook.f. & Arb & DC 1463 & FSE \\
\hline & Manettia cordifolia Mart. $^{\circ}$ & Tre & DC 1460 & FSE \\
\hline & Randia nitida (Kunth) DC. & Arb/Arv & DC 1923 & FSE \\
\hline & Rudgea jasminoides (Cham.) Müll.Arg. & Arb/Arv & DC 1960 & FSE \\
\hline RUTACEAE & Zanthoxylum acuminatum (Sw.) Sw.* & Arv & DC 1931 & FSE \\
\hline \multirow[t]{3}{*}{ SALICACEAE } & Casearia obliqua Spreng. & Arv & DC 1914 & FSE \\
\hline & Casearia selloana Eichler & Arv & DC 1965 & FSE \\
\hline & Casearia sylvestris $\mathrm{Sw} .^{\circ}$ & Arv & JN 26 & FSE \\
\hline \multirow[t]{3}{*}{ SAPINDACEAE } & Allophylus racemosus $\mathrm{Sw}$. & Arb/Arv & DC 1984 & FSE \\
\hline & Averrhoidium gardnerianum Baill.*० & Arb/Arv & DC 1926 & FSE \\
\hline & Cupania oblongifolia Mart. & Arv & DC 1955 & FSE \\
\hline SAPOTACEAE & Pouteria gardneri (Mart. \& Miq.) Baehni & Arv & DC 1954 & FSE \\
\hline \multirow[t]{4}{*}{ SOLANACEAE } & Capsicum parvifolium Sendth & Arb/Arv & DC 1922 & FSE \\
\hline & Cestrum uniflora (Pohl) D.Don & Arb/Arv & DC 1967 & FSE \\
\hline & Solanum depauperatum Dun.*o & Arb & DC 1434 & FSE \\
\hline & Solanum sp. nov. ${ }^{\circ}$ & Arb & DC 1889 & FSE \\
\hline TURNERACEAE & 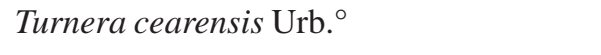 & Erv & DC 1444; LQ 1041 & CAA \\
\hline \multirow[t]{3}{*}{ URTICACEAE } & Laportea aestuans (L.) Chew & Erv & JN33 & FSE \\
\hline & Pilea hyalina Fenzl & Erv & JN 31 & FSE \\
\hline & Urera baccifera (L.) Gaudich. ex Wedd.*o & Arb/Arv & DC 1423, 1982 & FSE \\
\hline
\end{tabular}




\begin{tabular}{|c|c|c|c|c|}
\hline Família & Espécie & Hábito & Vouchers & Habitat \\
\hline \multirow[t]{3}{*}{ VERBENACEAE } & Aloysia virgata (Ruiz \& Pav.) Juss. ${ }^{\circ}$ & Arb/Arv & DC 1924; LQ 1004 & FSE \\
\hline & Lantana camara $\mathrm{L} .{ }^{* \circ}$ & Arb & LQ 1027 & FSE \\
\hline & Priva bahiensis A.DC. & Erv & $\begin{array}{l}\text { DC 1389; JN 34; } \\
\text { MF } 25\end{array}$ & FSE \\
\hline VIOLACEAE & Hybanthus verrucosus Paula-Souza & Erv & $\begin{array}{l}\text { DC } 1408,1429 \\
1903,1908\end{array}$ & FSE \\
\hline VISCACEAE & cf. Arceuthobium sp. & Erv & DC 1473 & FSE \\
\hline VITACEAE & Cissus albida Cambess $^{\circ}$ & Tre & DC 1894 & FSE \\
\hline
\end{tabular}

caatinga do Nordeste e as florestas decíduas do Brasil Central e norte de Mina Gerais; e (ii) o segundo formado com SB de 51\%, incluindo todas as fisionomias do Domínio da Floresta Atlântica. Dentro deste segundo agrupamento, aparece um grupo (grupo A) formado pelas duas áreas de Mata Atlântica de Pernambuco ("brejos de altitude") e a mata ciliar da Chapada Diamantina, e um outro grupo (grupo B) no qual podem ser distinguidos dois grandes grupos: grupo $\mathrm{C}$, essencialmente de florestas semidecíduas e grupo D, composto por florestas ombrófilas e semidecíduas do sul da Bahia e norte do Espírito Santo (grupo E) e florestas ombrófilas do Sudeste do Brasil (grupo F). A área de estudo do trabalho em questão (BAfsa) apareceu mais relacionada com a floresta semidecídua da Serra do Orobó (SB 89\%) dentro do grupo C.

\section{Discussão}

\section{Diversidade florística}

Devido à escassez de estudos florísticos dedicados ao levantamento de toda a flora de uma determinada região, a riqueza de espécies da vegetação florestal da SFR é aqui discutida e comparada com outros estudos baseandose principalmente no componente arbóreo.

O número de espécies arbóreas (81) da SFR foi superior ao número observado em diferentes fisionomias de caatinga do Nordeste (Araújo et al. 1998; Rodal et al. 1998; Rodal et al. 1999; Lemos \& Rodal 2002; Pereira et al. 2002; Alcoforado-Filho et al. 2003; Farias \& Castro 2004; Rocha et al. 2004; Amorim et al. 2005b; Andrade et al. 2005; Gomes et al. 2006; Lemos 2006; Fabricante \& Andrade
2007; Rodal et al. 2008), caatinga arbórea do norte de Minas Gerais (Santos et al. 2007; Santos et al. 2008) e floresta decídua sobre calcário do Brasil Central (Silva \& Scariot 2003, 2004a, b; Felfili et al. 2007) (Tab. 3).

Em diversos levantamentos realizados em Mata Atlântica s.l. (e.g. Moreno et al. 2003; Santos \& Kinoshita 2003; Silva \& Soares 2003; Andrade \& Rodal 2004; Guilherme et al. 2004; Meyer et al. 2004; Amorim et al. 2005a, 2008; Carvalho et al. 2005; Neves 2005; Pereira et al. 2006; Carvalho et al. 2007; Macedo 2007; Martini et al. 2007; Cardoso \& Queiroz 2008) a riqueza de espécies arbóreas tende a ser mais elevada do que a do presente estudo (Tab. 3). Porém, em outras áreas também de florestas estacionais ou ombrófilas, o valor encontrado foi inferior (Funch 1997; Sales et al. 1998; Ivanauskas \& Rodrigues 2000; Gomes et al. 2005; Rodal et al. 2005; Couto 2008; Souza 2008; Ribeiro-Filho et al. 2009) (Tab. 3). De modo geral, a análise da riqueza de espécies arbóreas sugere que o valor amostrado para a SFR está mais próximo dos valores de levantamentos em Mata Atlântica s.l. do que em caatinga e florestas secas do Brasil Central (Fig. 3). Ainda assim, é importante reconhecer que um maior número de espécies poderá ser encontrado na SFR, caso mais investimentos em coletas sejam realizados no futuro, o que possibilitaria até mesmo encontrar mais espécies inéditas, como as novas descobertas para a ciência aqui apresentadas. Por outro lado, deve ser destacado que de fato, uma estimativa da diversidade de espécies da SFR provavelmente não deverá superar em muito o valor aqui 


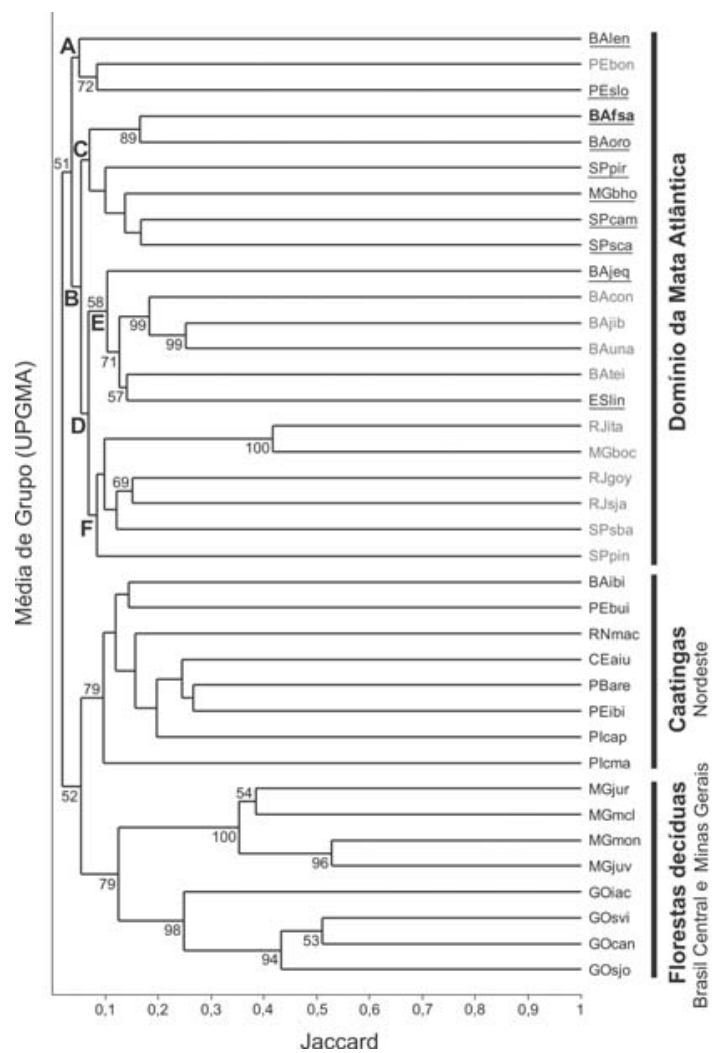

Figura 2 - Dendrograma de similaridade florística obtido a partir da análise de agrupamento (UPGMA) entre as 37 áreas representando diferentes fisionomias vegetacionais do Domínio da Mata Atlântica, da Caatinga e de florestas secas do Brasil Central. Os códigos referem-se às áreas listadas na Tabela 1. A área de estudo (BAfsa) aparece em negrito. As áreas grafadas em cinza são florestas ombrófilas e as áreas sublinhadas são florestas estacionais.

Figure 2 - Dendrogram of floristic similarity obtained from cluster analysis (UPGMA) among the 37 areas of different vegetation physiognomies of the Atlantic Forest domain, Caatinga, and dry forests of Central Brazil. Abbreviations refer to areas listed in Table 1. The abbreviation for the studied area (BAfsa) is in bold. The areas written in gray are moist forests and the underlined areas represent seasonal forests.

encontrado, levando-se em conta o histórico recente de queimadas observado em alguns locais.

Dentre as famílias mais diversas encontradas na SFR, Leguminosae, Euphorbiaceae e Malvaceae também são citadas como as famílias características de florestas tropicais sazonalmente secas (Prado 2000; Pennington et al. 2000), enquanto Myrtaceae e Orchidaceae apresentam maior diversidade em áreas de florestas úmidas do Domínio da Floresta Atlântica (Mori et al. 1983a, b). Aimportância da diversidade e endemismos de Myrtaceae na Mata Atlântica foi recentemente evidenciada no trabalho de Murray-Smith et al. (2008), que discutem a família como indicadora de dois grandes principais centros de diversidade de plantas: (i) Serra do Mar, desde o Paraná ao Rio de Janeiro e (ii) floresta costeira do norte do Espírito Santo e sul da Bahia. Esta separação também é evidente na análise de agrupamento realizada no presente estudo (Fig. 2).

A presença de três novas espécies reflete a necessidade de mais estudos nos fragmentos de florestas estacionais do estado da Bahia. Cardoso \& Queiroz (2008) também amostraram um considerável número de espécies importantes, incluindo dez novos táxons, novas ocorrências para a Bahia, além de coletas raras, em fragmentos de florestas estacionais localizados na Serra do Orobó, entre os municípios de Ruy Barbosa e Itaberaba, também próximos ao município de Feira de Santana.

\section{Relações fitogeográficas}

Devido à ocorrência marcante dos gêneros Cavanillesia (Malvaceae), Tabebuia s.l. (Bignoniaceae) e Aspidosperma (Apocynaceae), a vegetação florestal da SFR poderia ser incluída na Unidade I do domínio das caatingas, representada, segundo Andrade-Lima (1981), por áreas de vegetação arbórea estacional decidual (i.e. caatinga arbórea). Tal Unidade ocorre principalmente no norte de Minas Gerais e centro-sul da Bahia, geralmente sobre rochas calcárias ou cristalinas. Entretanto, a análise de similaridade mostrou que a SFR não está relacionada com a flora das caatingas ou das florestas decíduas sobre calcário do Brasil Central e norte de Minas Gerais. Estas, por sua vez, aparecem em um grande grupo separado do restante das fisionomias do Domínio da Floresta Atlântica. A ligação histórica entre as florestas secas do Brasil Central e a Caatinga, sugerida pelos padrões de distribuição de plantas (Prado 2000; Pennington et al. 2000, 2004), é também corroborada pela distribuição disjunta do lagarto Lygodactylus klugei na Caatinga e nos enclaves de florestas secas do vale do rio Paranã (Werneck \& Colli 2006). É interessante 
Tabela 3 - Comparação da riqueza de espécies, com destaque para o número de espécies representado pelo componente arbóreo, em diversos levantamentos florísticos e fitossociológicos realizados em Mata Atlântica s.l., Caatinga e floresta decídua do Brasil Central. Trabalhos que apresentam riqueza específica igual ao número de espécies arbóreas são aqueles que amostraram apenas este componente. Fisionomia: FO = floresta ombrófila, FS = floresta semidecídua, FD = floresta decídua, $\mathrm{FDC}=$ floresta decídua sobre afloramento calcário, $\mathrm{MC}=$ mata ciliar, $\mathrm{CAR}=$ caatinga arbórea, $\mathrm{CAA}=$ caatinga arbustiva sobre solo arenoso, $\mathrm{CAC}=$ caatinga arbustivo-arbórea sobre embasamento cristalino.

Table 3 - Comparison of total species richness and its number of trees in several floristic and phytosociological surveys carried out in Atlantic Forest s.l., Caatinga, and deciduous forest on limestone outcrop. Cited references showing species richness equal to the number of tree species indicate the works that surveyed only this component. Abbreviations for physiognomies: $\mathrm{FO}=$ moist forest, $\mathrm{FS}=$ semideciduous forest, $\mathrm{FD}=$ deciduous forest, $\mathrm{FDC}=$ deciduous forest on limestone outcrop, $\mathrm{MC}=$ gallery forest, $\mathrm{CAR}=$ arboreal caatinga, $\mathrm{CAA}=$ shrubby caatinga on sandy soil, $\mathrm{CAC}=$ treeshrubby caatinga on crystalline bedrock.

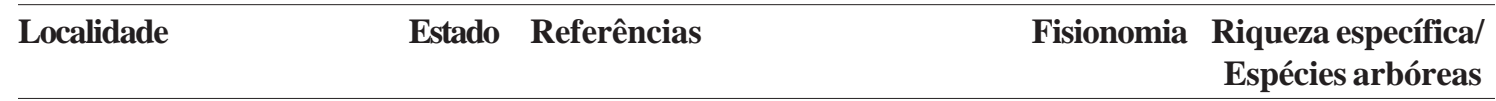

\section{MATA ATLÂNTICA s.l.}

\begin{tabular}{|c|c|c|c|c|}
\hline Feira de Santana & BA & Este estudo & FS & $173 / 81$ \\
\hline Jequié & BA & Macedo (2007) & FS & $594 / 275$ \\
\hline Uruçuca/Itacaré & BA & Martini et al. (2007) & FO & $144 / 144$ \\
\hline Ruy Barbosa & BA & Cardoso \& Queiroz (2008) & FS & $465 / 164$ \\
\hline Santa Terezinha & BA & Neves (2005) & $\mathrm{FO}$ & $353 / 353$ \\
\hline Una & BA & Amorim et al. (2008) & $\mathrm{FO}$ & $947 / 401$ \\
\hline Jussari & BA & Amorim et al. (2005a) & FO & $667 / 249$ \\
\hline Lençóis & BA & Ribeiro-Filho et al. (2009) & $\mathrm{MC}$ & $116 / 51$ \\
\hline Bonito & PE & Sales et al. (1998); Rodal et al. (2005) & $\mathrm{FO}$ & $217 / 53$ \\
\hline São Lourenço da Mata & PE & Andrade \& Rodal (2004) & FS & $85 / 85$ \\
\hline Belo Horizonte & MG & Meyer et al. (2004) & $\mathrm{MC}$ & $97 / 97$ \\
\hline Bocaina de Minas & MG & Carvalho et al. (2005) & $\mathrm{FO}$ & $221 / 221$ \\
\hline Itatiaia & $\mathrm{RJ} / \mathrm{MG}$ & Pereira et al. (2006) & FO & $444 / 444$ \\
\hline Bonito & RJ & Carvalho et al. (2007) & FO & $106 / 106$ \\
\hline Campos dos Goytacazes & RJ & Moreno et al. (2003) & FO & $210 / 210$ \\
\hline Piracicaba & $\mathrm{SP}$ & Ivanauskas \& Rodrigues (2000) & FD & $110 / 54$ \\
\hline Campinas & $\mathrm{SP}$ & Santos \& Kinoshita (2003) & FS & $175 / 162$ \\
\hline São Carlos & $\mathrm{SP}$ & Silva \& Soares (2003) & FS & $146 / 146$ \\
\hline Pindamonhangaba & SP & Gomes et al. (2005) & $\mathrm{FO}$ & $75 / 75$ \\
\hline Sete Barras & $\mathrm{SP}$ & Guilherme et al. (2004) & FO & $172 / 172$ \\
\hline \multicolumn{5}{|l|}{ CAATINGA } \\
\hline Ibiraba & BA & Rocha et al. (2004) & CAA & $86 / 23$ \\
\hline Novo Oriente & $\mathrm{CE}$ & Araújo et al. (1998) & CAA & $184 / 33$ \\
\hline Aiuaba & $\mathrm{CE}$ & Lemos (2006) & CAC & $161 / 66$ \\
\hline Juvenília & MG & Santos et al. (2008) & CAR & $44 / 44$ \\
\hline Montalvânia & MG & Santos et al. (2007) & CAR & $34 / 34$ \\
\hline $\begin{array}{l}\text { Areia e Remígio, } \\
\text { Agreste Paraibano }\end{array}$ & $\mathrm{PB}$ & Pereira et al. (2002) & CAA & $54 / 54$ \\
\hline Santa Luzia & PB & Fabricante \& Andrade (2007) & CAC & $22 / 22$ \\
\hline
\end{tabular}




\begin{tabular}{|c|c|c|c|c|}
\hline Localidade & Estado & Referências & Fisionomia & $\begin{array}{l}\text { Riqueza específica/ } \\
\text { Espécies arbóreas }\end{array}$ \\
\hline São João do Cariri & PB & Andrade et al. (2005) & CAC & $16 / 16$ \\
\hline Caruaru & PE & Alcoforado-Filho et al. (2003) & CAC & $96 / 55$ \\
\hline Buíque & PE & Gomes et al. (2006) & CAA & $192 / 29$ \\
\hline Buíque & PE & Rodal et al. (1998) & CAA & $35 / 35$ \\
\hline Ibimirim & PE & Rodal et al. (1999) & CAA & $139 / 26$ \\
\hline Floresta e Betânia & PE & Rodal et al. (2008) & CAC & $28 / 28$ \\
\hline Serra da Capivara & PI & Lemos \& Rodal (2002) & CAA & $56 / 56$ \\
\hline Campo Maior & PI & Farias \& Castro (2004) & CAA & $57 / 57$ \\
\hline Serra Negra do Norte & $\mathrm{RN}$ & Amorim et al. (2005b) & CAC & $15 / 15$ \\
\hline \multicolumn{5}{|l|}{ BRASIL CENTRAL } \\
\hline Iaciara & GO & Felfili et al. (2007) & FDC & $32 / 32$ \\
\hline $\begin{array}{l}\text { São Domingos, Faz. } \\
\text { Canadá }\end{array}$ & GO & Silva \& Scariot (2004a) & FDC & $48 / 48$ \\
\hline $\begin{array}{l}\text { São Domingos, Faz. } \\
\text { São Vicente }\end{array}$ & GO & Silva \& Scariot (2004b) & FDC & $51 / 51$ \\
\hline $\begin{array}{l}\text { São Domingos, Faz. } \\
\text { São José }\end{array}$ & GO & Silva \& Scariot (2003) & FDC & $36 / 36$ \\
\hline
\end{tabular}

notar que na análise de similaridade as áreas de calcário do Brasil Central e Minas Gerais foram agrupadas separadamente das demais caatingas do Nordeste. Alguns autores têm enfatizado que a vegetação decídua sobre solos litólicos de origem calcária possui fisionomia e composição florística próprias, incluindo diversos táxons endêmicos, e sugerem que as mesmas não deveriam ser tratadas meramente como uma fisionomia empobrecida, derivada da flora das florestas secas que ocorrem em outros tipos de solo (Silva \& Scariot 2003, 2004a, b; Pérez-García \& Meave 2004; Scariot \& Sevilha 2005; Pérez-García et al. 2009). A singularidade das florestas sobre calcário foi também recentemente confirmada por Santos (2009), em uma análise fitogeográfica ampla, comparando 179 áreas de florestas tropicais sazonalmente secas do leste do Brasil.

Alguns autores (e.g. Rizzini 1997; Veloso et al. 1991) preferem denominar as florestas altas de caatinga da Unidade I sensu Andrade-Lima (1981) como florestas estacionais. Essas florestas estacionais ocorrem em faixas descontínuas, tendo sido caracterizadas fitogeograficamente como uma estreita faixa de transição entre a Floresta Atlântica e as caatingas (Melo \& Rodal
2003). No entanto, Oliveira-Filho \& Fontes (2000), através de padrões de distribuição de espécies arbóreas, e Oliveira-Filho et al. (2005, 2006) analisando a similaridade florística entre diversas áreas do leste do Brasil propuseram que as florestas estacionais deveriam fazer parte do Domínio da Floresta Atlântica. Essa sugestão foi avaliada por Rodal et al. (2008), através de análise de similaridade entre as florestas estacionais do Nordeste situadas ao longo do Planalto da Borborema. Estes autores demonstraram que tais florestas estão relacionadas floristicamente não somente com a Floresta Atlântica Litorânea, como também com a vegetação xerofítica da Caatinga.

A presença das espécies lenhosas Aspidosperma pyrifolium (Apocynaceae), Casearia sylvestris (Salicaceae), Celtis iguanaea (Cannabaceae), Cordia trichotoma (Boraginaceae), Eugenia florida (Myrtaceae), Guapira opposita (Nyctaginaceae), Guazuma ulmifolia (Malvaceae), Machaerium acutifolium, Peltophorum dubium (Leguminosae), Maclura tinctoria (Moraceae), Maprounea guianensis, Sebastiania brasiliensis (Euphorbiaceae) e Urera baccifera (Urticaceae), indica que a SFR provavelmente está mais 


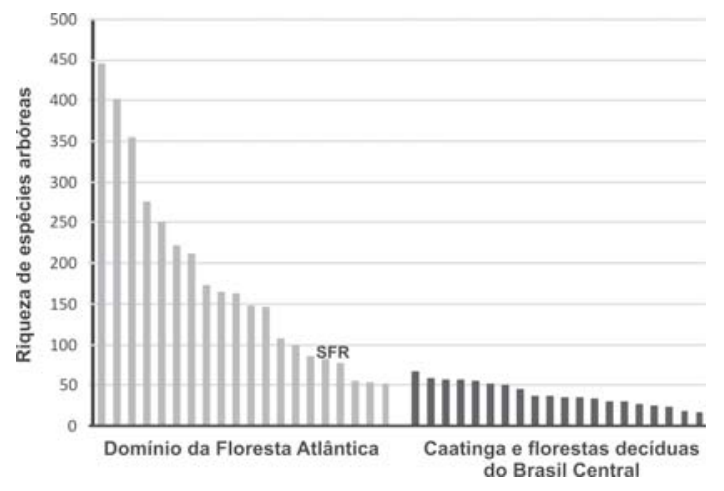

Figura 3 - Padrão de riqueza de espécies arbóreas em diferentes fitofisionomias de Mata Atlântica e florestas tropicais sazonalmente secas (Caatinga e florestas decíduas do Brasil Central). Os dados foram obtidos a partir dos levantamentos listados na Tabela 3. A área de estudo (SFR) aparece em destaque dentro da variação de riqueza de espécies para a Mata Atlântica.

Figure 3 - Pattern of tree species richness in distinct vegetation physiognomies of Atlantic Forest and seasonally dry tropical forests (Caatinga and deciduous forests on limestone outcrop of Central Brazil). Data were obtained from surveys listed in Table 3. The studied area (SFR) is among the variation of species richness in Atlantic Forest.

relacionada floristicamente com o Domínio da Floresta Atlântica do que outras áreas de caatinga da Região Nordeste do Brasil. Essas espécies estão entre as 100 que foram listadas por OliveiraFilho et al. (2006) como características das florestas sazonalmente secas tropicais dentro do núcleo florístico da Floresta Atlântica s.l. (Oliveira-Filho \& Fontes 2000). A diversidade florística encontrada na SFR, superior ao comumente encontrado em áreas de caatinga, bem como a análise de similaridade, confirmam a classificação da SFR como floresta estacional relacionada com a Floresta Atlântica. A análise das outras formas de vida também reforça essa classificação. Quando comparamos a lista de espécies do presente estudo com a da Reserva do Teimoso, um fragmento de Floresta Atlântica no sul da Bahia (Amorim et al. 2005a) várias delas (38 spp.), incluindo árvores, arbustos, epífitas ou ervas, também foram encontradas na SFR, a exemplo de: Actinostemon appendiculatus, Cnidoscolus oligandrus (Euphorbiaceae), Alseis floribunda, Faramea hyacinthina, (Rubiaceae), Aspidosperma parvifolium, Brasiliopuntia brasiliensis (Cactaceae),
Capparis frondosa (Cruciferae), Cavanillesia arborea (Malvaceae), Coccoloba declinata (Polygonaceae), Dichorisandra hexandra (Commelinaceae), Erythroxylum petrae-cabralli (Erythroxylaceae), Ficus nymphaeifolia (Moraceae), Gallesia integrifolia (Phytolaccaceae), Goniorrhachis marginata, Pseudopiptadenia bahiana (Leguminosae), Sinningia barbata (Gesneriaceae), Solanum depauperatum (Solanaceae), Tabebuia roseoalba (Bignoniaceae) e Zanthoxylum acuminatum (Rutaceae). Cardoso \& Queiroz (2008) também chegaram a uma conclusão similar para a vegetação florestal da Serra do Orobó, a qual, em termos de composição de espécies, apresenta maior similaridade com outras áreas de Floresta Atlântica (sensu Oliveira-Filho \& Fontes 2000) do que a vegetação de caatinga. De fato, a SFR apresentou maior similaridade florística com a Serra do Orobó, em um grupo (C) de Mata Atlântica composto essencialmente por florestas estacionais. O outro grupo (D) formado pela grande maioria das florestas ombrófilas do norte do Espírito Santo e sul da Bahia em um subgrupo (E) e do Sudeste do Brasil em outro (D) é fortemente correlacionando com o padrão de diversidade e endemismos de espécies de Myrtaceae nestas regiões (Murray-Smith et al. 2008). A separação entre florestas estacionais semidecíduas e ombrófilas também foi observada por Ferraz et al. (2004), através de análise de similaridade, e por Nascimento (2009), através de análise de parcimônia de endemismos, entre diversas áreas de Mata Atlântica do Nordeste. Além disso, estes autores verificaram, assim como no presente estudo, que os enclaves de florestas úmidas (ou brejos de altitude) situados dentro dos limites do Bioma Caatinga devem igualmente fazer parte do Domínio da Floresta Atlântica.

Como discutido amplamente ao longo deste trabalho, a questão de que o Domínio da Floresta Atlântica deveria incluir também as florestas estacionais semidecíduas parece estar bem resolvida através de diferentes fontes de evidência (Oliveira-Filho \& Fontes 2000; Ferraz et al. 2004; Amorim et al. 2005a; Oliveira-Filho et al. 2006; Cardoso \& Queiroz 2008; Rodal et 
al. 2008; Nascimento 2009). O desafio agora seria a proposta de um novo sistema de classificação que pudesse incluir os avanços obtidos com estes estudos. Para isso, um novo sistema deveria também uniformizar toda a pletora de nomes (e.g. mata seca, floresta seca, caatinga arbórea, floresta decídua, mata de cipó, entre outros) comumente usada na literatura para descrever as florestas estacionais. Neste sentido, Oliveira-Filho (2009) propõe um novo sistema de nomenclatura e classificação para a vegetação da América do Sul tropical e subtropical a leste dos Andes, incluindo o Domínio da Floresta Atlântica, baseado principalmente em aspectos fisionômicoecológicos e sem deixar de utilizar a base do sistema de classificação do IBGE. Usando os cinco atributos hierárquicos desta nova proposta de classificação, a fitofisionomia florestal da SFR é definida como "floresta latifoliolada estacional semideciduifólia tropical submontana crassissólica granítica".

\section{Conclusões}

A composição florística das florestas estacionais neotropicais é ainda muito pouco estudada, quando comparada aos estudos desenvolvidos em florestas úmidas (Prado 2000; Pennington et al. 2000, 2006; SánchezAzofeifa et al. 2005; Espírito-Santo et al. 2006). Além disso, as florestas estacionais estão sendo continuamente fragmentadas, como resultado de fortes pressões antrópicas ao longo dos últimos dois séculos (Janzen 1988; Werneck et al. 2000; Sánchez-Azofeifa et al. 2005; Scariot \& Sevilha 2005; Espírito-Santo et al. 2006, 2008, 2009; Miles et al. 2006). Os estudos e esforços para conservação das áreas de florestas estacionais no estado da Bahia também são escassos, não diferindo muito da realidade observada no restante do país e no mundo (Sánchez-Azofeifa et al. 2005; EspíritoSanto et al. 2006, 2008; Miles et al. 2006). Com a realização do presente estudo foi possível acessar um pouco mais do conhecimento da flora desse tipo de vegetação.

A presença de três novas espécies nos gêneros Neomarica, Pseudobombax e Solanum mostra a importância da vegetação florestal da SFR no que diz respeito à conservação de sua flora, bem como a necessidade de estudos adicionais na região. As queimadas frequentes na SFR podem se tornar uma preocupação alarmante, uma vez que espécies de colonização favorecida pelo fogo geralmente provocam uma perda da diversidade local (Toniato \& Oliveira-Filho 2004; Grund et al. 2005). Diante dessa crescente pressão antrópica, a continuidade dos estudos poderá subsidiar futuras estratégias de conservação e uso sustentável dos recursos naturais disponíveis.

Baseando-se na diversidade florística, análise de similaridade e distribuição de algumas espécies, foi possível observar que a SFR está mais relacionada floristicamente ao Domínio da Floresta Atlântica do que às caatingas. Neste sentido, a SFR deve ser entendida como um subconjunto das florestas úmidas, sendo a sua sazonalidade possivelmente relacionada a um gradiente de umidade.

\section{Agradecimentos}

Os autores agradecem ao Sr. Manoel pelo apoio logístico durante as visitas de campo na SFR; aos funcionários do HUEFS pela disposição na organização da coleção da SFR; aos dois revisores anônimos pelas críticas e valiosas sugestões ao manuscrito; e aos seguintes especialistas pela colaboração na confirmação e/ou identificação de algumas espécies: Ana Luiza Côrtes (Acanthaceae), Silvana Ferreira (Asteraceae), Marlon Machado (Cactaceae), Lidiane Aona (Commelinaceae), Daniela Carneiro-Torres (Euphorbiaceae), André Gil (Iridaceae), Leslie Landrum (Myrtaceae), Marla Ibrahim (Myrtaceae), Cássio van den Berg (Orchidaceae), Reyjane P. Oliveira (Poaceae), Efigênia de Melo (Polygonaceae).

\section{REFERÊNCIAS BIBLIOGRÁFICAS}

Ab'Saber, A.N. 1974. O domínio morfoclimático semiárido das caatingas brasileiras. Série Geomorfologia, 43. USP, São Paulo. 37p.

Alcoforado-Filho, F.G.; Sampaio, E.V.S.B. \& Rodal, M.J.N. 2003. Florística e fitossociologia de um remanescente de vegetação caducifólia 
espinhosa arbórea em Caruaru, Pernambuco. Acta Botanica Brasilica 17(2): 287-303.

Amorim, A.M.; Jardim, J.G.; Fiaschi, P.; Clifton, B.C.; Carvalho, A.M.V. \& Thomas, W.W. 2005a. The vascular plants of a forest fragment in Southern Bahia, Brazil. Sida 21(3): 1727-1752.

Amorim, A.M.; Thomas, W.W.; Carvalho, A.M. \& Jardim, J.G. 2008. Floristics of the Una Biological Reserve, Bahia, Brasil. Memoirs of the New York Botanical Garden 100: 67-146.

Amorim, I.L.; Sampaio, E.V.S.B. \& Araújo, E.L. 2005b. Flora e estrutura da vegetação arbustivo-arbórea de uma área de caatinga do Seridó, RN, Brasil. Acta Botanica Brasilica 19(3): 615-623.

Andrade, K.V.S.A. \& Rodal, M.J.N. 2004. Fisionomia e estrutura de um remanescente de floresta estacional semidecidual de terras baixas no nordeste do Brasil. Revista Brasileira de Botânica 27(3): 463-474.

Andrade, L.A.; Pereira, I.M.; Leite, U.T. \& Barbosa, M.R.V. 2005. Análise da cobertura de duas fitofisionomias de caatinga, com diferentes históricos de uso, no município de São João do Cariri, estado da Paraíba. Cerne 11(3): 253-262.

Andrade-Lima, D. 1981. The caatingas dominium. Revista Brasileira de Botânica 4: 149-153.

APG II - Angiosperm Phylogeny Group. 2003. An update of the angiosperm phylogeny group classification for the orders and families of flowering plants. Botanical Journal of the Linnean Society 141: 399-436.

Araújo, F. S.; Sampaio, E. V. S. B.; Figueiredo, M. A.; Rodal,M.J.N.\&Fernandes, A.G. 1998.Composição florística da vegetação de carrasco, Novo Oriente, CE. Revista Brasileira de Botânica 21(2): 105-116.

BAHIA. 2006. SEI (Superintendência de Estudos Econômicose Sociais da Bahia.). www.sei.ba.gov.br. Acesso em 10 de outubro de 2006.

Cardoso, D.B.O.S. \& Queiroz, L.P. 2008. Floristic composition of seasonally dry tropical forest fragments in Central Bahia, Northeastern Brazil. Journal of the Botanical Research Institute of Texas 2(1): 551-573.

Carvalho, D.A.; Oliveira-Filho, A.T.; van den Berg, E.; Fontes, M.A.L.; Vilela, E.A.; Marques, J.J.G.S.M. \& Carvalho, W.A.C. 2005. Variações florísticas e estruturais do componente arbóreo de uma floresta ombrófila alto-montana às margens do Rio Grande, Bocaina de Minas, MG, Brasil. Acta Botanica Brasilica 19: 91-109.

Carvalho, F.A.; Nascimento, M.T. \& Braga, J.M.A. 2006. Composiçãoe riqueza florística do componente arbóreo da Floresta Atlântica submontana na região de Imbaú, município de Silva Jardim, RJ. Acta Botanica Brasilica 20(3): 727-740.

Carvalho, F.A.; Nascimento, M.T. \& Braga, J.M.A. 2007. Estrutura e composição florística do estrato arbóreo de um remanescente de Mata Atlântica submontana no município de Rio Bonito, RJ, Brasil (Mata Rio Vermelho). Revista Árvore 31(4): 717-730.

Carvalho-Sobrinho, J.G. \& Queiroz, L.P. 2005. Composição florística de um fragmento de Mata Atlântica na serra da Jibóia, Santa Terezinha, Bahia, Brasil. Sitientibus Série Ciências Biológicas 5(1): 20-28.

Cestaro, L.A. \& Soares, J.J. 2004 . Variações florística e estrutural e relações fitogeográficas de um fragmento de floresta decídua no Rio Grande do Norte, Brasil. Acta Botanica Brasilica 18(2): 203-218.

Conceição, A.A.; Pirani, J.R. \& Meirelles, S.T. 2007. Floristics, structure and soil of insular vegetation in four quartzite-sandstone outcrops of "Chapada Diamantina", Northeast Brazil. Revista Brasileira de Botânica 30(4): 641-656.

Couto, A.P.L. 2008. Florística e estrutura de uma floresta submontana no Parque Nacional da Chapada Diamantina, Bahia, Brasil. Dissertação de Mestrado. Universidade Estadual de Feira de Santana, Feira de Santana. 51p.

Espírito-Santo, M.M.; Fagundes, M.; Nunes, Y.R.F.; Fernandes, G.W.; Sánchez-Azofeifa, G.A. \& Quesada, M. 2006. Bases para a conservação e uso sustentável das florestas estacionais deciduais brasileiras: a necessidade de estudos multidisciplinares. Unimontes Científica 8: 13-22.

Espírito-Santo, M.M.; Sevilha, A.C.; Scariot, A.; Sánchez-Azofeifa, G.A.; Noronha, S.E. 2008. Florestas estacionais deciduais brasileiras: distribuição e estado de conservação. MG-Biota: Boletim Técnico da Diretoria e Biodiversidade do IEF-MG 1(2): 5-13.

Espírito-Santo, M.M.; Sevilha, A.C.; Anaya, F.C.; Barbosa, R.; Fernandes, G.W.; Sánchez-Azofeifa, G.A.; Scariot, A.; Noronha, S.E. \& Sampaio, C.A. 2009. Sustainability of tropical dry forests: two case studies in Southeastern and Central Brazil. Forest Ecology and Management 258(6): 922-930.

Fabricante, J.R. \& Andrade, L.A. 2007. Análise estrutural de um remanescente de caatinga no Seridó paraibano. Oecologia Brasiliensis 11(3): 341-349.

Farias, R.R.S. \& Castro, A.A.J.F. 2004. Fitossociologia de trechos da vegetação do Complexo de Campo 
Maior, Campo Maior, PI, Brasil. Acta Botanica Brasilica 18(4): 949-963.

Felfili, J.M.; Nascimento, A.R.T.; Fagg, C.W. \& Meirelles, E.M. 2007. Floristic composition and community structure of a seasonally deciduous forest on limestone outcrops in Central Brazil. Revista Brasileira de Botânica 30(4): 611-621.

Ferraz, E.B.N.; Araújo, E.L. \& Silva, S.I.. 2004. Floristic similarities between lowland and montane areas of Atlantic Coastal Forest in Northeastern Brazil. Plant Ecology 174: 59-70.

França, F.; Melo, E. \& Santos, C. C. dos. 1997. Flora de inselbergs da região de Milagres, Bahia, Brasil: I. Caracterização da vegetação e lista de espécies de dois inselbergs. Sitientibus 17: 163-184.

Funch, L.S. 1997. Composição florística e fenologia de mata ciliar e mata de encosta adjacentes ao Rio Lençóis, Lençóis, BA. Tese de Doutorado. Universidade Estadual de Campinas, Campinas. 298p.

Funch, L.S. 2008. Florestas da região norte do Parque Nacional da Chapada Diamantina e seu entorno. In: Funch, L.S.; Funch, R.R. \& Queiroz, L.P. (eds.). Serra do Sincorá, Parque Nacional da Chapada Diamantina. Radami Editora Gráfica, Feira de Santana. Pp. 63-77.

Funch,L.S.; Funch, R.R.; Harley, R.M.; Giulietti, A.M.; Queiroz, L.P.; França, F.; Melo, E.; Gonçalves, C.N. \& Santos, T. R. 2005. Florestas estacionais semideciduais. In: Juncá, F.A.; Funch, L. \& Rocha, W. (eds.). Biodiversidade e conservação da Chapada Diamantina. Ministério do Meio Ambiente, Brasília. Pp. 181-193.

Funch, L.S.; Rodal, M.J.N. \& Funch, R. 2008. Floristic aspects of forests of the Chapada Diamantina, Bahia, Brazil. Memoirs of the New York Botanical Garden 100: 193-220.

Gomes, A.P.S.; Rodal, M.J.N. \& Melo, A.L. 2006. Florística e fitogeografia da vegetação arbustiva subcaducifólia da Chapada de São José, Buíque, PE, Brasil. Acta Botanica Brasilica 20(1): 37-48.

Gomes, E.P.C.; Fisch, S.T.V. \& Mantovani, W. 2005. Estrutura e composição do componente arbóreo na Reserva Ecológica do Trabiju, Pindamonhangaba, SP, Brasil. Acta Botanica Brasilica 19(3): 451-464.

Grund, K.; Conederab, M.; Schrödera, H. \& Waltherc, G.R. 2005. The role of fire in the invasion process of evergreen broad-leaved species. Basic and Applied Ecology 6: 47-56.

Guilherme, F.A.G.; Morellato, P.C. \& Assis, M.A. 2004. Horizontal and vertical tree community structure in a lowland Atlantic Rain Forest, Southeastern Brazil. Revista Brasileira de Botânica 27(4): 725-737.

Hammer, Ø.; Harper,D.A.T. \& Ryan, D.T. 2001.PAST: palaeontological statistics software package for education and data analysis. Palaeontologia Electronica 4(1): 1-9.

Ivanauskas, N.M. \& Rodrigues, R.R. 2000. Florística e fitossociologia de remanescentes de floresta estacional decidual em Piracicaba, São Paulo, Brasil. Revista Brasileira de Botânica 23(3): 291-304.

Janzen, D.H. 1988. Tropical dry forests. The most endangered major tropical ecosystem. In: Wilson, E.O. (ed.). Biodiversity. National Academy Press, Washington. Pp. 130-137.

Lemos, J.R. 2004. Composição florística do Parque Nacional Serra da Capivara, Piauí, Brasil. Rodriguésia 55(85): 55-66.

Lemos, J. R. 2006. Florística, estrutura e mapeamento da vegetação da Estação Ecológica de Aiuaba, Ceará. Tese de Doutorado. Universidade de São Paulo, São Paulo. 139p.

Lemos, J.R. \& Rodal, M.J.N. 2002. Fitossociologia do componente lenhoso de um trecho da vegetação de caatinga no Parque Nacional Serra da Capivara, Piaú, Brasil. Acta Botanica Brasilica 16(1): 23-42.

Macedo, G.E.L. 2007. Composição florística e estrutura do componente arbóreo-lianescente de um trecho de floresta estacional semidecidual no município de Jequié, Bahia, Brasil. Dissertação de Mestrado. Universidade Federal Rural de Pernambuco, Recife. 102p.

Martini, A.M.Z.; Fiaschi, P.; Amorim, A.M. \& Paixão, J.L. 2007. A hot-point within a hot-spot: a high diversity site in Brazil's Atlantic Forest. Biodiversity and Conservation 16: 3111-3128.

Melo, J.I.M. \& Rodal, M.J.N. 2003. Levantamento florístico de um trecho de floresta serrana no planalto de Garanhuns, estado de Pernambuco. Acta Scientiarum: Biological Sciences 25(1): 173-178.

Meyer, S.T.; Silva, A.F.; Marco Jr., P. \& Meira-Neto, J.A.A. 2004. Composição florística da vegetação arbórea de um trecho de floresta de galeria do Parque Estadual do Rola-Moça na Região Metropolitana de Belo Horizonte, MG, Brasil. Acta Botanica Brasilica 18(4): 701-709.

Miles, L.; Newton, A.C.; DeFries, R.S.; Ravilious, C.; May, I.; Blyth, S.; Kapos, V. \& Gordon, J.E. 2006. A global overview of the conservation status of tropical dry forests. Journal of Biogeography 33: 491-505. 
Moreno, M.R.; Nascimento, M.T. \& Kurtz, B.C. 2003. Estrutura e composição florística do estrato arbóreo em duas zonas altitudinais na Mata Atlântica de encosta da região do Imbé, RJ. Acta Botanica Brasilica 17: 371-386.

Mori, S.A.; Boom, B.M.; Carvalho, A.M. \& Santos, T.S. 1983a. Ecological importance of Myrtaceae in an eastern Brazilian wet forest. Brittonia 15(1): 68-70.

Mori, S.A.; Boom, B.M.; Carvalho, A.M. \& Santos, T. S. 1983b. Southern Bahian moist forests. Botanical Review 49: 155-232.

Mori, S.A.; Mattos-Silva, L.A.; Lisboa, G. \& Coradin, L. 1985. Manual de manejo do herbário fanerogâmico. Centro de Pesquisas do Cacau, Ilhéus. 97p.

Murphy, F.G. \& Lugo, A.E. 1986. Ecology of tropical dry forest. Annual Review of Ecology and Systematics 17: 67-88.

Nascimento, F.H.F. 2009. As florestas alto montanas nordestinas, sul da Chapada Diamantina, Bahia: florística, estrutura e relações biogeográficas. Tese de Doutorado. Universidade Estadual de Feira de Santana, Feira de Santana. 250p.

Neves, M.L.C. 2005. Caracterização da vegetação de um trecho de Mata Atlântica de encosta na Serra da Jibóia, Bahia. Dissertação de Mestrado. Universidade Estadual de Feira de Santana, Feira de Santana. 101p.

Oliveira-Filho, A.T.2009. Classificaçãodas fitofisionomias da América do Sul cisandina tropical e subtropical: proposta de um novo sistema - prático e flexível - ou uma injeção a mais de caos? Rodriguésia 60(2): 237-258.

Oliveira-Filho, A.T. \& Fontes, A.L. 2000. Patterns of floristic differentiation among Atlantic Forests in Southeastern Brazil and the influence of climate. Biotropica 32: 793-810.

Oliveira-Filho, A.T.; Tameirão-Neto, E.; Carvalho, W.A.C.; Werneck, M.; Brina, A.E.; Vidal, C.V.; Resende, S.C. \& Pereira, J.A.A. 2005. Análise florística do compartimento arbóreo de áreas de Floresta Atlântica sensu lato na região das Bacias do Leste (Bahia, Minas Gerais, Espírito Santo e Rio de Janeiro). Rodriguésia 56(87): 185-235.

Oliveira-Filho, A.T.; Jarenkow, J.A. \& Rodal, M.J.N. 2006. Floristic relationships of seasonally dry forests of eastern South America based on tree species distribution patterns. In: Pennington, R.T.; Lewis, G.P. \& Ratter, J.A. (eds.). Neotropical savannas and dry forests: plant diversity, biogeography, and conservation. Taylor \&
Francis CRC Press, Oxford. Pp. 59-192.

Pennington, R.T.; Prado, D.E. \& Pendry, C.A. 2000. Neotropical seasonally dry forests and Quaternary vegetation changes. Journal of Biogeography 27: 261-273.

Pennington, R.T.; Lavin, M.; Prado, D.E.; Pendry, C.A.; Pell, S.K. \& Butterworth, C.A. 2004. Historical climate change and speciation: neotropical seasonally dry forest plants show patterns of both Tertiary and Quaternary diversification. Philosophical Transactions of the Royal Society of London Series B, Biological Sciences 359: 515-538.

Pennington, R.T.; Lewis, G.P.; Ratter, J.A. 2006. An overview of the plant diversity, biogeography and conservation of neotropical savannas and seasonally dry forests. In: Pennington, R. T.; Lewis, G.P. \& Ratter, J.A. (eds.). Neotropical savannas and dry forests: plant diversity, biogeography, and conservation. Taylor \& Francis CRC Press, Oxford. Pp. 1-20.

Pereira, I.M.; Andrade,L.A.; Barbosa, M.R.V.\& Sampaio, E.V.S.B. 2002. Composição florística e análise fitossociológica do componente arbustivoarbóreo de um remanescente florestal no agreste paraibano. Acta Botanica Brasilica 16(3): 357-369.

Pereira, I.M.; Oliveira Filho, A.T.; Botelho, S.A.; Carvalho, W.A.C.; Fontes, M.A.L.; Schiavini, I. \& Silva, A.F. 2006. Composição florística do compartimento arbóreo de cinco remanescentes florestais do maciço do Itatiaia, Minas Gerais e Rio de Janeiro. Rodriguésia 57: 103-126.

Pérez-García, E.A. \& Meave, J.A. 2004. Heterogeneity of xerophytic vegetation of limestone outcrops in a tropical deciduous forest region. Plant Ecology 175: 147-163.

Pérez-García, E.A.; Sevilha, A.C.; Meave, J.A. \& Scariot, A. 2009. Floristic differentiation in limestone outcrops of Southern Mexico and Central Brazil: a beta diversity approach. Boletín de la Sociedad Botánica de México 84: 45-58.

Prado, D.E. 2000. Seasonally dry forests of tropical South America: from forgotten ecosystems to a new phytogeographic unit. Edinburgh Journal of Botany 57: 437-461.

Prado, D.E. 2003. As caatingas do Brasil. In: Leal, I.R.; Tabarelli, M. \& Silva, J.M.C. (eds.). Ecologia e conservação da Caatinga. Ed. Universidade Federal de Pernambuco, Recife. Pp. 3-73.

Prado, D.E. \& Gibbs, P.E. 1993. Patterns of species distributions in the dry seasonal forests of South 
America. Annals of the Missouri Botanical Garden 80: 902-927.

Queiroz, L.P. 2006. The Brazilian Caatinga: phytogeographical patterns inferred from distribution data of the Leguminosae. In: Pennington, R.T.; Lewis, G.P. \& Ratter, J.A. (eds.). Neotropical savannas and dry forests: plant diversity, biogeography, and conservation. Taylor \& Francis CRC Press, Oxford. Pp. 113-149.

Ribeiro-Filho, A.A. 2009. Composição florística da floresta ciliar do rio Mandassaia, Parque Nacional da Chapada Diamantina, Bahia, Brasil. Rodriguésia 60(2): 265-276.

Rizzini, C.T. 1997. Tratado de fitogeografia do Brasil. Âmbito Cultural Ed., Rio de Janeiro. 747p.

Rocha, P.L.B.; Queiroz, L.P. \& Pirani, J.R. 2004. Plant species and habitat structure in a sand dune field in the Brazilian Caatinga: a homogenous habitat harbouring an endemic biota. Revista Brasileira de Botânica 27(4): 739-755.

Rodal, M.J.N. \& Nascimento, L.M. 2002. Levantamento florístico da floresta serrana da Reserva Biológica de Serra Negra, microrregião de Itaparica, Pernambuco, Brasil. Acta Botanica Brasilica 16(4): 481-500.

Rodal, M.J.N. \& Nascimento, L.M. 2006. The arboreal component of a dry forest in northeastern Brazil. Brazilian Journal of Biology 66(2A): 479-491.

Rodal, M.J.N. \& Sampaio, E.V.S.B. 2002. A vegetação do bioma caatinga. In: Sampaio, E.V.S.B.; Giulietti, A.M.; Virgínio, J. \& Gamarra-Rojas, C.F.L. (eds.). Vegetação e flora da Caatinga. APNE/CNIP, Recife. Pp. 11-24.

Rodal, M.J.N.; Andrade, K.V.A.; Sales, M.F. \& Gomes, A.P.S. 1998. Fitossociologia do componente lenhoso de um refúgio vegetacional no município de Buíque, Pernambuco. Revista Brasileira de Botânica 58(3): 517-526.

Rodal, M.J.N.; Nascimento, L.M.; Melo, A.L. 1999. Composição florística de um trecho de vegetação arbustiva caducifólia, no município de Ibimirim, Pernambuco, Brasil. Acta Botanica Brasilica 13(1): 14-29.

Rodal, M.J.N.; Sales, M.F.; Silva, M.J. \& Silva, A.G. 2005. Flora de um brejo de altitude na escarpa oriental do planalto da Borborema, PE, Brasil. Acta Botanica Brasilica 19(4): 843-858.

Rodal,M.J.N.; Barbosa, M.R.V.\& Thomas, W.W. 2008. Do the seasonal forests in northeastern Brazil represent a single floristic unit? Brazilian Journal of Biology 68(3): 467-475.

Rodal, M.J.N.; Costa, K.C.C. \& Silva, A.C.B.L. 2008. Estrutura da vegetação caducifólia espinhosa
(Caatinga) de uma área do sertão central de Pernambuco. Hoehnea 35(2): 209-217.

Rolim, S.G.; Ivanauskas, N.M.; Rodrigues, R.R.; Nascimento, M.T.; Gomes, J.M.L.; Folli, D.A. \& Couto, H.T.Z. 2006. Composição florística do estrato arbóreo da floresta estacional semidecidual na planície aluvial do rio Doce, Linhares, ES, Brasil. Acta Botanica Brasilica 20(3): 549-561.

Sales, M. F.; Mayo, S. J. \& Rodal, M. J. N. 1998. Plantas vasculares das florestas serranas de Pernambuco: um checklist da flora ameaçada dos Brejos de Altitude. Imprensa Universitária da Universidade Federal Rural de Pernambuco, Recife. 130p.

Sambuichi, R.H.R. 2002. Fitossociologia e diversidade de espécies arbóreas em cabruca (Mata Atlântica raleada sobre plantação de cacau) na região Sul da Bahia, Brasil. Acta Botanica Brasilica 16(1): 89-101.

Sánchez-Azofeifa, G.A.; Kalácska, M.; Quesada, M.; Calvo-Alvarado, J.C.; Nassar, J.M. \& Rodrigues, J.P. 2005. Need for integrated research for a sustainable future in tropical dry forests. Conservation Biology 19: 285-286.

Santos, K. \& Kinoshita, L.S. 2003. Flora arbustivoarbórea do fragmento de floresta estacional semidecidual do Ribeirão Cachoeira, município de Campinas, SP. Acta Botanica Brasilica 17(3): 325-341.

Santos, R.M. 2009. Identidade e relações florísticas da caatinga arbórea do norte de Minas Gerais e sudeste da Bahia. Tese de Doutorado. Universidade Federal de Lavras, Lavras. 118p.

Santos, R.M.; Vieira, F.A.; Fagundes, M.; Nunes, Y.R.F. \& Gusmão, E. 2007. Riqueza e similaridade florística de oito remanescentes florestais no norte de Minas Gerais, Brasil. Revista Árvore 31(1): 135-144.

Santos, R.M.; Vieira, F.A.; Santos, P.F.; Morais, V.M.; Medeiros, M.A. 2008. Estrutura e florística de um remanescente florestal na fazenda Ribeirão, município de Juvenília, MG, Brasil. Revista Caatinga 21(4): 154-162.

Scariot A. \& Sevilha A.C. 2005. Biodiversidade, estrutura e conservação de florestas estacionais deciduais no cerrado. In: Scariot A.; SousaSilva, J.C. \& Felfili, J.M. (eds.). Ecologia, biodiversidade e conservação do Cerrado, Ministério do Meio Ambiente, Brasília. Pp. 121-139.

Silva, L.A. \& Soares, J.J. 2003. Composição florística de um fragmento de floresta estacional semidecídua no município de São Carlos-SP. Revista Árvore 27(5): 647-656. 
Silva, L.A. \& Scariot, A. 2003. Composição florística e estrutura da comunidade arbórea em uma floresta estacional decidual em afloramento calcário (Fazenda São José, São Domingos, GO, bacia do rio Paranã). Acta Botanica Brasilica 17(2):305-313.

Silva, L. A. \& Scariot, A. 2004a. Comunidade arbórea de uma floresta estacional decídua sobre afloramento calcário na bacia do rio Paraná. Revista Árvore 28(1): 61-67.

Silva, L.A. \& Scariot, A. 2004b. Composição e estrutura da comunidade arbórea de uma floresta estacional decidual sobre afloramento calcário no Brasil Central. Revista Árvore 28(1): 69-75.

Soares-Filho, A.O. 2000. Estudo fitossociológico em duas florestas em região ecotonal no planalto de Vitória da Conquista, Bahia, Brasil. Dissertação de Mestrado. Universidade de São Paulo, São Paulo. $147 \mathrm{p}$.

Souza, A. E. A. 2008. Composição florística e estrutura de uma mata de encosta, Serra da Bacia, Parque Nacional da Chapada Diamantina, Bahia, Brasil. Dissertação de Mestrado. Universidade Estadual de Feira de Santana, Feira de Santana. 66p.

Stannard, B.L. (ed.). 1995. Flora of the Pico das Almas, Chapada Diamantina, Bahia, Brazil. Royal Botanic Gardens, Kew. 877p.

Stradmann, M.T.S. 1997. Composição florística de um trecho da mata ciliar da trilha do Bodão e estudo comparativo do estrato arbustivoarbóreo, Rio Ribeirão, Parque Nacional da Chapada Diamantina, Bahia, Brasil. Trabalho de conclusão do Curso de Recursos Ambientais. Universidade Federal da Bahia, Salvador. 69p.

Stradmann, M.T.S. 2000. Composição florística da mata ciliar da foz do rio Capivara e análise quantitativa do estrato arbustivo-arbóreo, Parque Nacional da Chapada Diamantina. Dissertação de Mestrado. Universidade Federal da Bahia, Salvador. 130p.

Tavares, S.; Paiva, F.A.F; Carvalho, G.H. \& Tavares, E.J.S. 1979. Inventário florestal no estado da Bahia, I - Resultados de um inventário florestal nos municípios de Una, Porto Seguro, Santa Cruz de Cabrália, Prado, Itamaraju, Belmonte e Ilhéus.
Recursos Vegetais no. 9, Superintendência do Desenvolvimento do Nordeste, Departamento de Recursos Naturais, Recife. 234p.

Thomas, W.W.; Jardim, J.G.; Fiaschi, P.; MarianoNeto, E. \& Amorim, A.M.A. 2009. Composição florística e estrutura do componente arbóreo de uma área transicional de Floresta Atlântica no Sul da Bahia, Brasil. Revista Brasileira de Botânica 32: 41-54.

Toniato, M.T.Z. \& Oliveira-Filho, A.T. 2004. Variations in tree community composition and structure in a fragment of tropical semideciduous forests in southeastern Brazil related to different human disturbance histories. Forest Ecology and Management 198: 319-339.

Velloso, A.L.; Sampaio, E.V.S.B.; Giulietti, A.M.; Barbosa, M.R.V.; Castro, A.A.J.F.; Queiroz, L.P.; Fernandes, A.; Oren, D.C.; Cestaro, L.A.; Carvalho, A.J.E.; Pareyn, F.G.C.; Silva, F.B.R.; Miranda, E.E.; Keel, S. \& Gondim, R.S. 2002. Ecorregiões: propostas para o Bioma Caatinga. APNE, The Nature Conservancy do Brasil, Recife. 76p.

Veloso, H.P.; Rangel-Filho, A.L.R. \& Lima, J.C.A. 1991. Classificação da vegetação brasileira, adaptada a um sistema universal. Fundação Instituto Brasileiro de Geografia e Estatística, Rio de Janeiro. 123p.

Werneck, F.P. \& Colli, G.R. 2006. The lizard assemblage from seasonally dry tropical forest enclaves in the Cerrado biome, Brazil, and its association with the Pleistocenic Arc. Journal of Biogeography 33: 1983-1992.

Werneck, M.S.; Franceschinelli, E.V. \& TameirãoNeto, E. 2000. Mudanças na florística e estrutura de uma floresta decidual durante um período de quatro anos (1994-1998), na região do Triângulo Mineiro, MG. Revista Brasileira de Botânica 23(4): 401-413.

Zappi, D.C.; Lucas, E.; Stannard, B.L.; Lughadha, E.N.; Pirani, J.R.; Queiroz, L.P.; Atkins, S.; Hind, D.J.N.; Giulietti, A.M.; Harley, R.M. \& Carvalho, A.M. 2003. Lista das plantas vasculares de Catolés, Chapada Diamantina. Boletim de Botânica da USP 21(2): 345-39. 